\title{
Long-period High-amplitude Red Variables in the KELT Survey
}

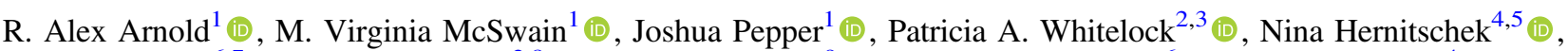

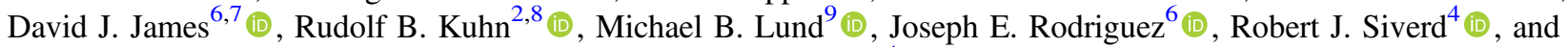 \\ Keivan G. Stassun ${ }^{4}$ (D) \\ ${ }^{1}$ Lehigh University, Department of Physics, 16 Memorial Drive East, Bethlehem, PA, 18015, USA \\ ${ }^{2}$ South African Astronomical Observatory, P.O. Box 9, Observatory, 7935 Cape Town, South Africa \\ ${ }^{3}$ Department of Astronomy, University of Cape Town, 7701 Rondebosch, South Africa \\ ${ }^{4}$ Department of Physics \& Astronomy, Vanderbilt University, 6301 Stevenson Center Lane, Nashville, TN 37235, USA \\ 5 Data Science Institute, Vanderbilt University, 2414 Highland Avenue, Nashville, TN 37212, USA \\ ${ }^{6}$ Center for Astrophysics|Harvard \& Smithsonian, 60 Garden Street, Cambridge, MA 02138, USA \\ ${ }_{8}^{7}$ Black Hole Initiative at Harvard University, 20 Garden Street, Cambridge, MA 02138, USA \\ ${ }^{8}$ Southern African Large Telescope, P.O. Box 9, Observatory, 7935, Cape Town, South Africa \\ ${ }^{9}$ Caltech/IPAC-NExScI, Pasadena, CA 91125, USA \\ Received 2019 June 25; revised 2019 December 20; accepted 2020 January 13; published 2020 March 16
}

\begin{abstract}
We present a sample of 4132 Mira-like variables (red variables with long periods and high amplitudes) in the Kilodegree Extremely Little Telescope (KELT) survey. Of these, 376 are new Mira-like detections. We used Two Micron All Sky Survey (2MASS) colors to identify candidate asymptotic giant branch stars. We searched for photometric variability among the candidate asymptotic giant branch stars and identified stars that show periodic variability. We selected variables with high amplitudes and strong periodic behavior using a Random Forest classifier. Of the sample of 4132 Mira-like variables, we estimate that $70 \%$ are Miras and 30\% are semiregular (SR) variables. We also adopt the method of using $\left(W_{R P}-W_{K_{s}}\right)$ versus $\left(J-K_{S}\right)$ colors in distinguishing between O-rich and C-rich Miras and find it to be an improvement over 2MASS colors.
\end{abstract}

Unified Astronomy Thesaurus concepts: Mira variable stars (1066); Long period variable stars (935); Time series analysis (1916)

Supporting material: machine-readable table

\section{Introduction}

Late-type, low- to intermediate-mass red giant stars are almost always unstable against pulsation and form a class of variable stars known as the long-period variables (LPVs). Of these, Miras are perhaps the best known. Miras are a short-lived phase of a low- to intermediate-mass star's life when it has evolved to the tip of the asymptotic giant branch (AGB). At this stage, these stars exhibit large bolometric luminosities, and this, combined with their low average densities and high-amplitude pulsations, causes them to experience significant mass loss through stellar winds before they meet their eventual fate as white dwarfs surrounded by transient planetary nebulae. This process enriches the interstellar medium with heavy elements, in particular carbon and s-process elements, and therefore the chemistry of Miras directly influences the composition of stars and the overall chemical evolution of galaxies. Aside from their importance to Galactic chemistry, Miras also show promise as distance indicators. Multiple studies (Feast et al. 1989; Hughes \& Wood 1990; Whitelock et al. 2008; and for a general overview see Whitelock 2012 and references therein) have found evidence for a period-luminosity (PL) relation in the near-infrared for Miras. In the future they may offer a viable alternative to Cepheids as extragalactic standard candles (Feast et al. 2002; Whitelock 2013; Huang et al. 2018).

Miras are conventionally defined to have periods $>100$ days and amplitudes of $\Delta V>2.5 \mathrm{mag}$ (Payne-Gaposchkin 1951; Samus' et al. 2017), but their amplitudes decrease at longer wavelengths with typical amplitudes of $\Delta I>0.8$ (Soszyński et al. 2009) and $\Delta K>0.4$ mag (Whitelock et al. 2006). While an amplitude criterion can effectively identify Miras, the specific amplitude limits are somewhat arbitrary (Whitelock 1996a).
Another class of LPVs are the semiregular variables (SRs). They are less studied than Miras and are defined to have amplitudes of $\Delta V<2.5$ (Payne-Gaposchkin 1951; Samus' et al. 2017). SR variables represent a more heterogeneous class than Miras and consist of stars on both the AGB and red giant branch (RGB) with a range of masses (Whitelock 1996b). Their light curves typically exhibit more irregular behavior than Miras, with some exhibiting signs of multiperiodicity (Cadmus 2015). Despite what their name implies, SRs can exhibit regular periodic behavior similar to Miras but with smaller amplitudes. This distinguishing amplitude criterion between Miras and SRs is arbitrary, and it is possible that low-amplitude Miras and highamplitude SRs may share physical similarities (Kerschbaum \& Hron 1992; Bedding \& Zijlstra 1998). We should note that even the condition of regular periodic behavior is arbitrary since Miras themselves are not necessarily regular pulsators and can exhibit cycle-to-cycle amplitude changes (Whitelock 1996b). In particular, carbon-rich Mira variables experience asymmetric mass loss that causes irregularities in their light curves (e.g., see the visual light curve of R For in Figure 6 of Whitelock et al. 1997). Observations irregularly spaced in time can also obscure the regularity of Mira and SR light curves, making such a distinction difficult. For these reasons, we will attempt to discuss high-amplitude, long-period red variable stars without discriminating between Miras and SRs with near-regular periodicity. We will refer to these stars of interest as Mira-like variables.

The cause of variability in Miras and SRs is radial pulsation, which occurs in either fundamental or overtone modes. An important discovery by Wood et al. (1999) showed that LPVs, which include Miras and SRs, populate multiple PL sequences. Recent studies (Trabucchi et al. 2017 and references therein) 
have determined that these sequences are due to the dominant pulsating mode present in the star, with Miras corresponding to fundamental mode pulsation. Most SRs are overtone pulsators, but Bedding \& Zijlstra (1998) found that a majority of their SR sample fell on the same PL sequence as Miras. These studies suggest that a more physically meaningful distinction between classes of LPVs would be the dominant mode of the star's pulsation instead of an amplitude cutoff, but mode determination is difficult without information on both their periods and luminosities. We do not attempt to pursue this method of classification in this work, but it may be worth future analysis. Sources with luminosities determined in future work can be used for refining PL relations, and comparisons between observed and modeled pulsation modes can serve as tests of stellar interior models.

Because of their high luminosity and scientific value, many catalogs of LPVs have been produced by sky surveys such as those from the MACHO project (Alcock et al. 1993; Wood et al. 1999), the All-Sky Automated Survey (ASAS; Pojmanski 2002; Vogt et al. 2016), the Northern Sky Variability Survey (NSVS; Woźniak et al. 2004a, 2004b), the Optical Gravitational Lensing Experiment (Udalski et al. 1992; Soszyński et al. 2009, 2011, 2013), and most recently Gaia (Gaia Collaboration et al. 2016, 2018; Mowlavi et al. 2018). The Kilodegree Extremely Little Telescope (KELT) provides advantages over several previous surveys owing to its high photometric precision, its long time baseline of observations, and its coverage of both the northern and southern sky. It also detects bright stars that make for easier follow-up observations.

We have created a catalog of 4132 Mira-like variables from the KELT survey. These are high-amplitude, long-period red variables that are predominantly Miras. Section 2 discusses the KELT data and the cross-match between KELT and the Two Micron All Sky Survey (2MASS). Our method for creating our catalog is presented in Section 3. In Section 4 we discuss results of our catalog and compare it to similar catalogs such as that of Vogt et al. (2016). Section 5 discusses a technique of classifying Miras as oxygen- or carbon-rich based on the analysis of Lebzelter et al. (2018). We summarize these results in Section 6.

\section{Observations and Data Reduction}

\subsection{Observations}

The observational data used in this study are time-series photometry taken from the KELT survey (Pepper et al. 2007, 2012). KELT is a photometric survey consisting of two smallaperture $(42 \mathrm{~mm})$, wide-field $\left(26^{\circ} \times 26^{\circ}\right)$ telescopes originally designed to search for transiting planets orbiting bright stars with $8<V<13$ and began operations in 2005. The KELT survey uses an effective passband roughly equivalent to a very broad $R$-band filter. The survey performs observations in both the northern and southern sky, with the northern telescope at Winer Observatory in Arizona, United States, and the southern telescope at the South African Astronomical Observatory near Sutherland, South Africa. With these two locations, KELT observes over $70 \%$ of the sky. The time baselines of KELT observations vary, with some of the longest up to $10 \mathrm{yr}$. These long baselines, combined with a typical cadence of 30 minutes and high photometric precision, result in the KELT survey being a valuable tool for studying phenomena beyond exoplanet detection.
One point of consideration is the large pixel scale of the KELT survey $\left(\sim 23^{\prime \prime}\right)$, which can result in stars in the KELT image blending together. This blending between a target star and nearby neighbors can cause two possible effects: variability from the neighboring sources appearing in the light curve of a target, and contaminating sources diluting the variability of the target star. The latter effect dampens the amplitude of the magnitude variations and flattens the light curve minima (see 2MASS J08312641-5414231 in Figure 1). Because the variables we are interested in are typically brighter than nearby neighbors, and the high-amplitude longperiod variability of Miras is not typically seen in other variable stars, we assume that the variability is attributed to the target star in almost all cases. Due to the high amplitudes of Miras, any dilution must be significant to affect our ability to detect Miras. We discuss attempts to identify and correct for blending in Section 4.1.

When stars fade to a brightness below the background sky, our ability to obtain reliable differential photometry becomes dominated by systematic noise, and the pipeline-generated light curves display unphysically deep features. Since the point where that occurs depends on the sky background, degree of nearby star blending, point-spread function (PSF) size and shape, and pixel quantum efficiency, we have been unable to implement an automated cut to remove such behavior. Some of the Miras in our sample show this effect at their photometric minima (such as 2MASS J02355221-6235005 in Figure 1), but we have not seen any cases where this effect causes spurious detections in our sample.

Due to the German Equatorial Mount used by KELT, photometric data for a target star are observed in separate eastern and western orientations. The data for each orientation are reduced separately, resulting in most KELT objects having two light curves, but there do exist KELT objects only identified in one orientation. When necessary, we have created combined light curves for the KELT objects by combining their eastern and western data. A magnitude offset typically exists between the two orientations that must be determined for each object before creating the combined light curve. We calculated this magnitude offset for each KELT object by first dividing the eastern and western light curves into identical 1 day bins. We determined the median magnitude of each bin and then calculated the differences of the eastern and western medians for each day. The median of these differences is the eastern/ western offset. After applying the offset correction for each KELT object, the eastern and western light curves were combined into one light curve. A photometric error of $0.1 \mathrm{mag}$ was assumed for all photometric data.

The KELT data are divided into fields based on sky location. There are 23 fields observed by the northern telescope and 24 by the southern telescope. Fields that have been in operation earlier, e.g., N01, have baselines nearing $10 \mathrm{yr}$, while fields that have begun operations more recently, e.g., N16, have shorter time baselines between 1 and 4 yr. Because Miras have long periods, typically 100-1000 days, we removed KELT fields with baselines of observations less than $2.5 \mathrm{yr}$ from our study (Figure 2).

\subsection{Cross-matching KELT to $2 M A S S$}

To determine the 2MASS photometry of each source, we cross-matched the list of KELT stars to the 2MASS catalog 

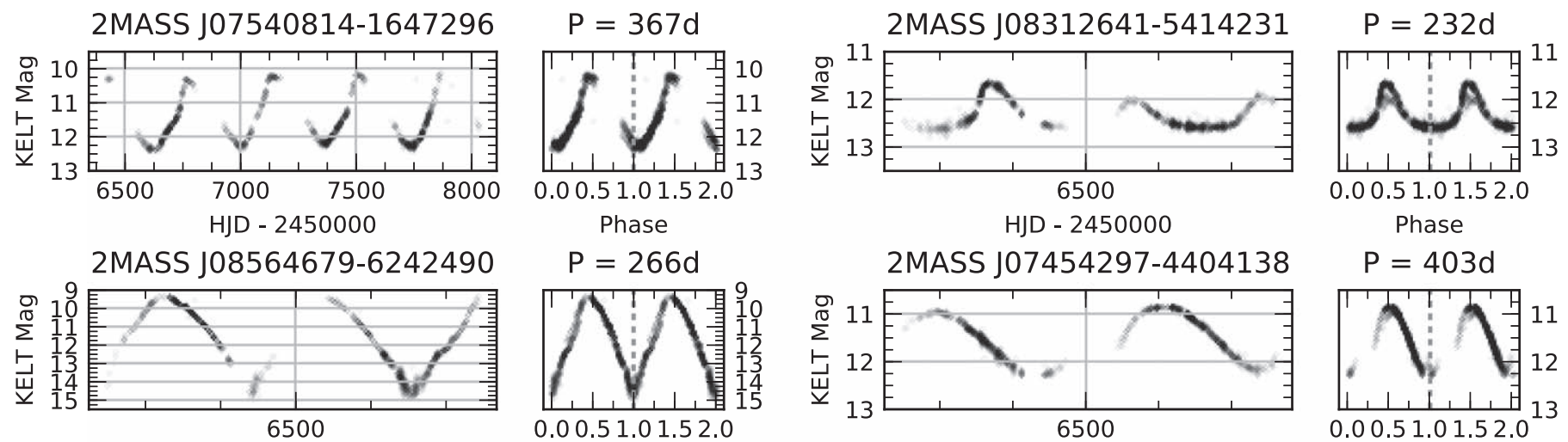

$P=266 d$

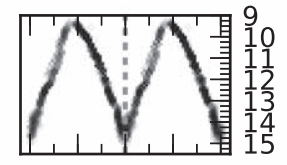

0.00 .51 .01 .52 .0

HJD - 2450000

Phase

2MASS J16043612-2406006

$P=163 d$
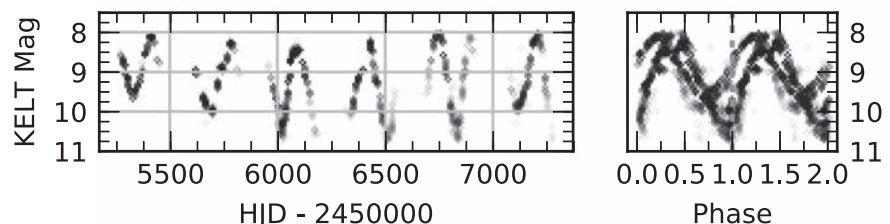

Phase

2MASS J19152147+0233020

$P=481 d$
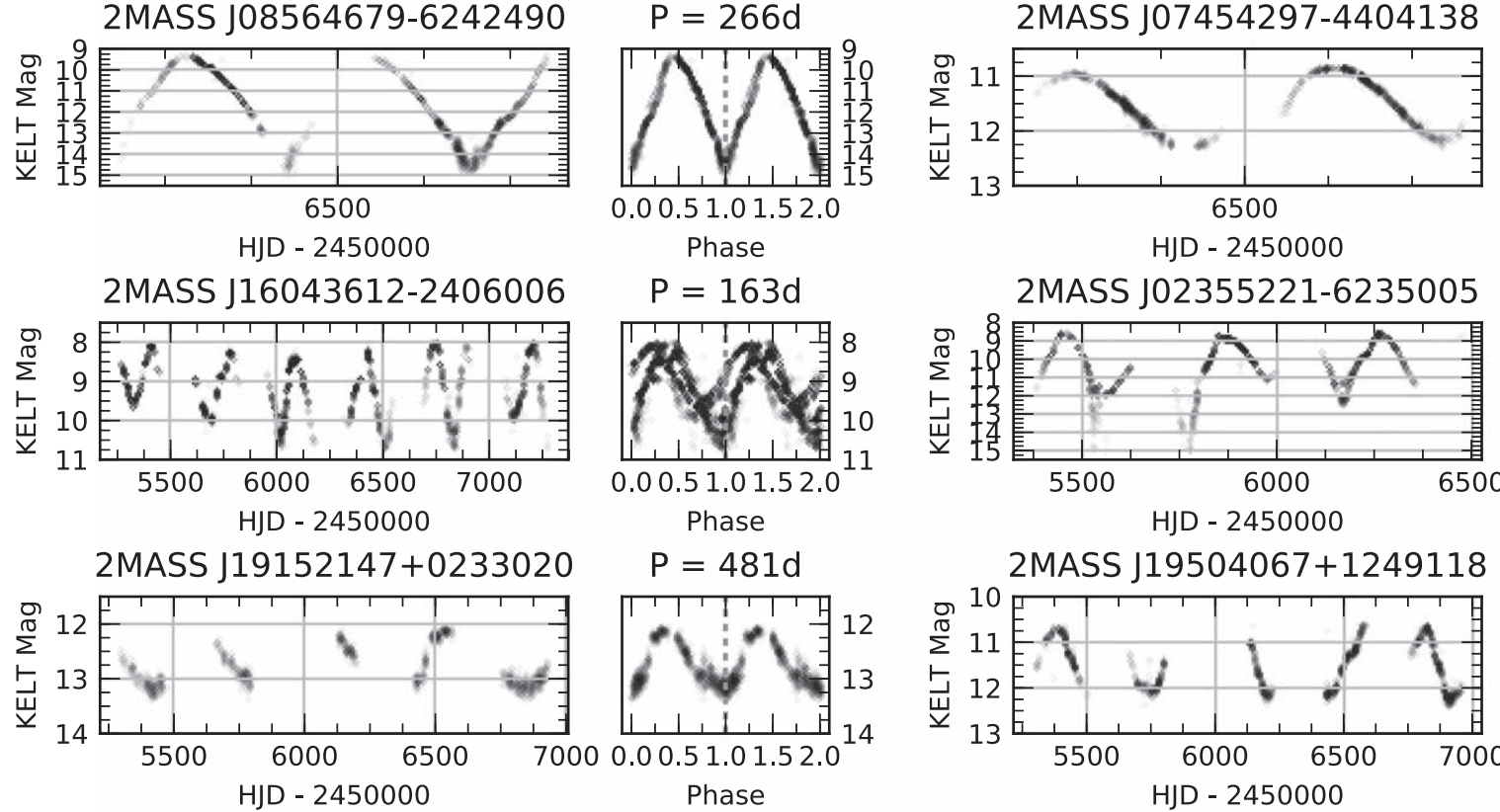

$P=403 d$
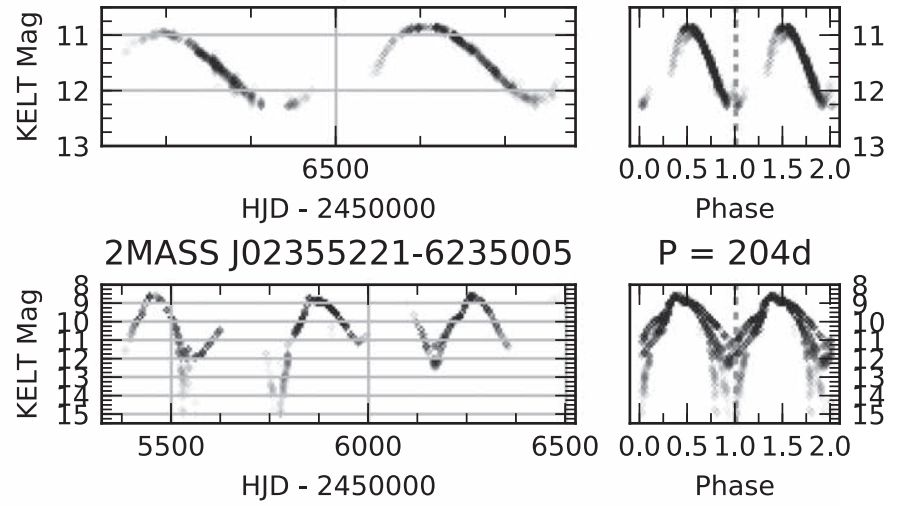

Phase

$P=204 d$

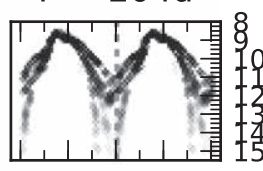

2MASS J19504067+1249118

Phase

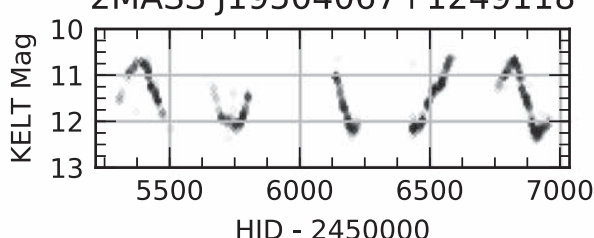

$P=237 d$

2MASS J18091982-0901054

$P=208 d$
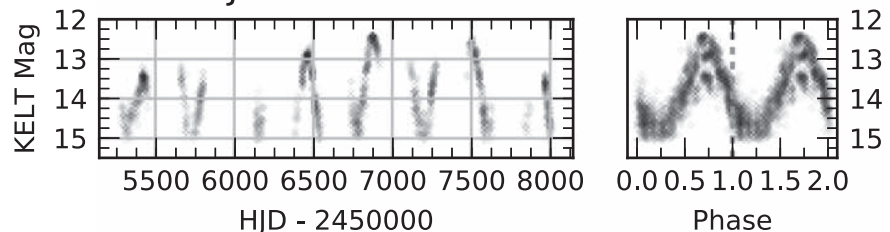

0.00 .51 .01 .52 .0

Phase

$P=419 d$

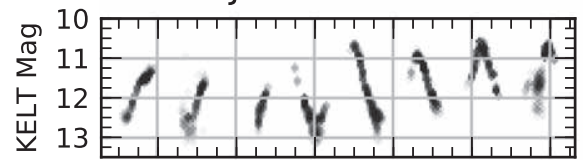

550060006500700075008000

HJD - 2450000

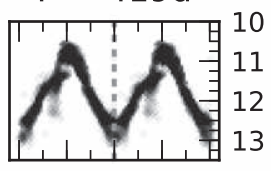

0.00 .51 .01 .52 .0

Phase

2MASS J19233241+2147026

$P=248 d$
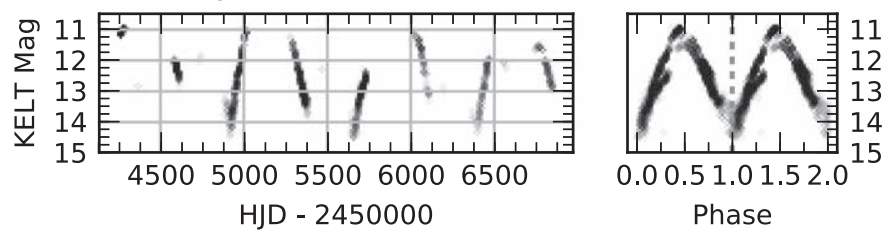

Phase
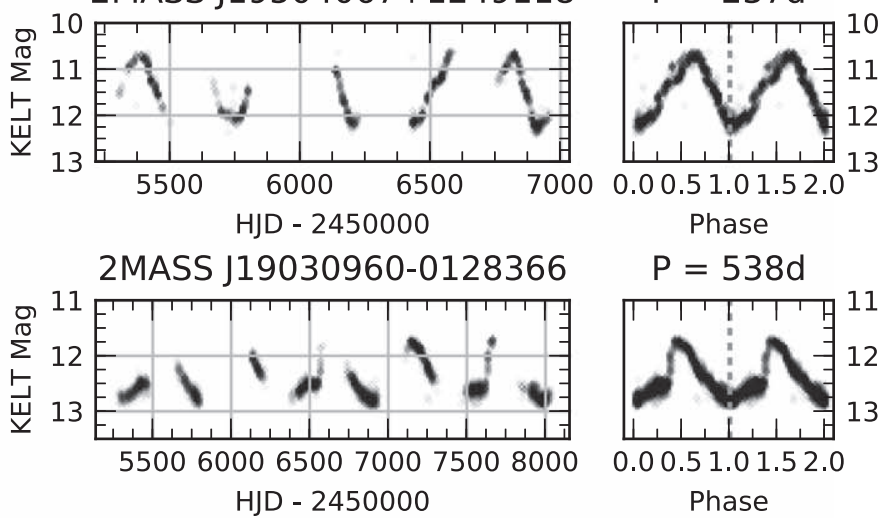

Phase

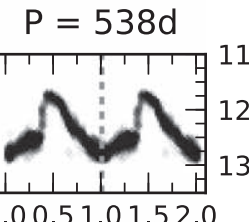

0.00 .51 .01 .52 .0

Phase

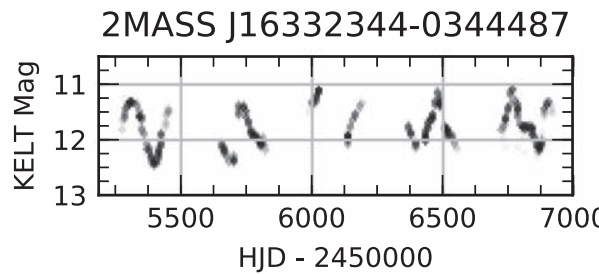

$P=145 d$

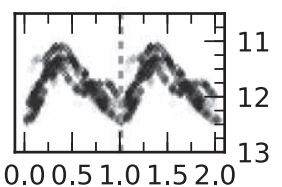

Phase
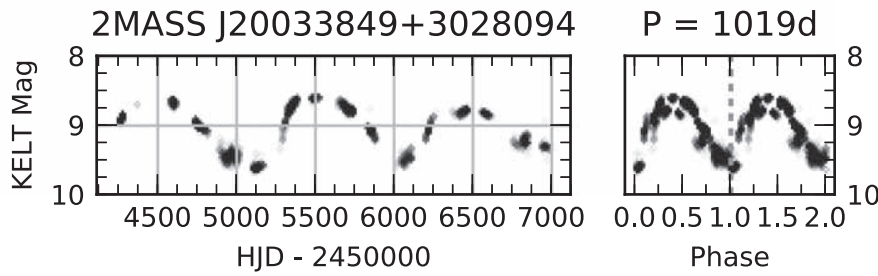

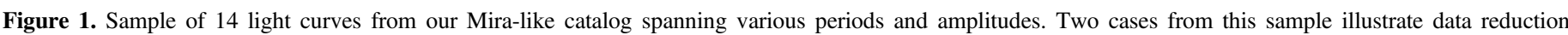

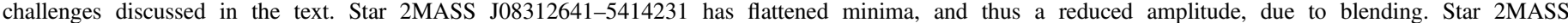

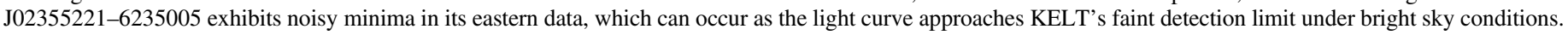

(Skrutskie et al. 2006) using a 25" search radius and considered only 2MASS objects with $J$ magnitude brighter than 16 . We chose this search radius because it is approximately the size of a KELT pixel, and smaller radii were found to exclude potential matches. This radius can result in multiple matches to 2MASS; therefore, we compared the KELT magnitude, $R_{\mathrm{KELT}}$, to $J$ for each 2MASS object in the search radius. We are interested in red variables, and therefore we excluded 2MASS objects with $\left(R_{\mathrm{KELT}}-J\right)<-1$. The 2 MASS object with the brightest $J$ magnitude was then chosen as the appropriate match. These search criteria were able to match $97 \%$ of the KELT catalog to a 2MASS object. Note that for the remainder of the paper we refer to the catalog of KELT objects that match to 2MASS as the KELT catalog. 


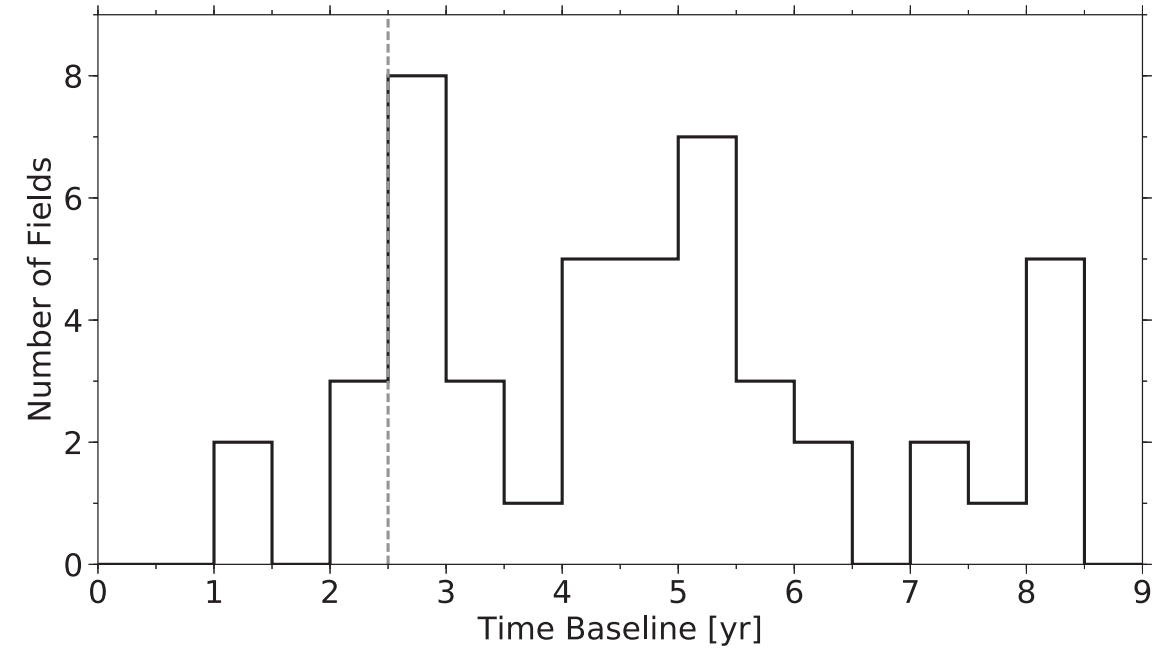

Figure 2. Maximum time baseline of the various KELT fields used in this study. KELT consists of older fields nearing almost 10 yr of observations and newer fields with baselines between 1 and 4 yr. For our study we removed fields with baselines less than $2.5 \mathrm{yr}$. This has been indicated by the gray dashed line.

\section{Methods}

\subsection{Preliminary Cuts for Selecting Variable Candidates}

To identify candidate AGB stars, we used a color-color diagram of $\left(H-K_{s}\right)$ versus $(J-H)$ (Feast et al. 1982). To justify our color cut criteria, we first selected a sample of known Miras and SRs from the International Variable Star Index (VSX) from the American Association of Variable Star Observers (AAVSO; Watson et al. 2006, using the 2019 version of the database). We then matched these to the KELT catalog using the matched 2MASS coordinates. We used the 2MASS colors of this matched VSX sample and inspected their color-color distribution to determine appropriate cuts. Figure 3 shows the $\left(H-K_{s}\right)$ versus $(J-H)$ colors of all KELT matches in gray and VSX Miras and SRs in red. We selected stars with $\left(H-K_{s}\right)>0.15$ and $(J-H)>0.7$ as candidate AGB stars. These cuts are shown by the dashed black lines in Figure 3.

After the candidate AGB stars were found, we searched for variability among these objects. We first identified candidate AGB objects with potential variability based on the light curve mean magnitude and standard deviation of the magnitudes. This allows us to quickly select for probable variable stars. We desired to reduce the number of objects under consideration before combining light curves. Therefore, we calculated the standard deviation of the eastern and western light curve magnitudes separately. Individually, an eastern or western light curve may not cover a full phase of variability; thus, we classified an object as a candidate variable if the standard deviation of either light curve lies above the line shown in Figure 4.

After the standard deviation cuts, we found cases where the standard deviation was inflated by outlying data points in the light curve. To account for these cases, we rejected stars with eastern and western light curve amplitudes less than $0.2 \mathrm{mag}$ after removing the $5 \%$ and $95 \%$ outlying data points. For the remainder of our analysis we removed only the $1 \%$ and $99 \%$ outlying data points so as to not remove data for the later period analysis. After applying these amplitude cuts, we also removed light curve data points with KELT magnitude fainter than 15 in order to remove noisy data likely below the faint detection limit of KELT. This magnitude cut is effective in removing spurious data, but we note that there still exists a sample of light curves

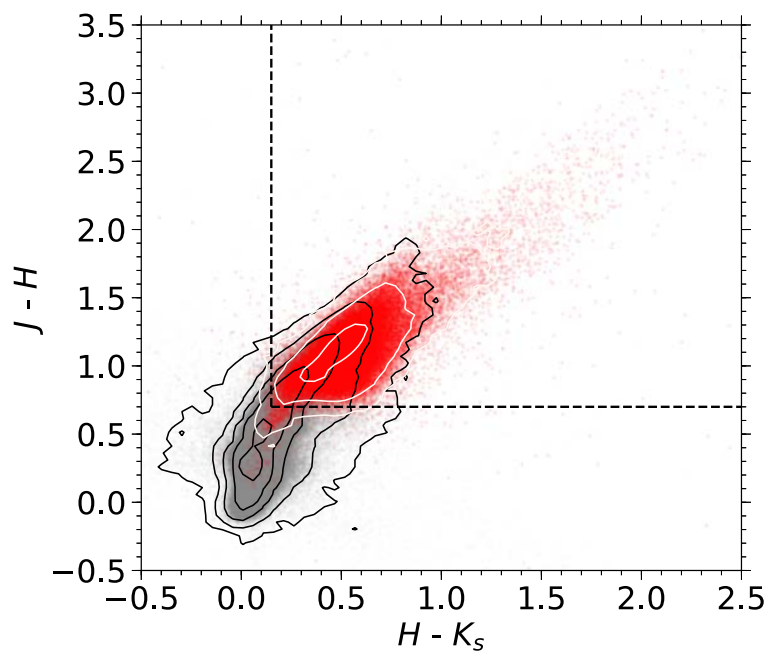

Figure 3. Color cuts of the KELT sample (gray) and the VSX Miras and SRs (red). Our color cuts of $\left(H-K_{s}\right)>0.15$ and $(J-H)>0.7$ are shown as dashed lines. Contours express the density of plotted data. The $H-K_{s}$ and $J-H$ data are divided into 0.05 mag bins, and the contours are logarithmically spaced to circumscribe bins containing 100-100,000 data points.

exhibiting noisy minima. We then combined eastern and western light curves using the method described in Section 2.1. Collectively, these cuts primarily remove nonred, nonvariable objects and allow us to greatly reduce the light curve data considered for further analysis by $98 \%$ (from 4.2 million KELT objects to 70,000$)$.

\subsection{Selecting Mira-like Variables with Random Forest}

We applied a Random Forest (RF) classifier (Breiman 2001) to identify Mira-like variables among objects passing the aforementioned cuts. RF is an ensemble machine-learning method that uses multiple decorrelated decision trees to predict the classification type of an object from various measurable properties (features) of the object. The decision trees "learn" the classification types using a training set of data containing features of objects with known classifications (labels). To decorrelate the decision trees, each is built on a random selection of the training data, and a random subset of the total 


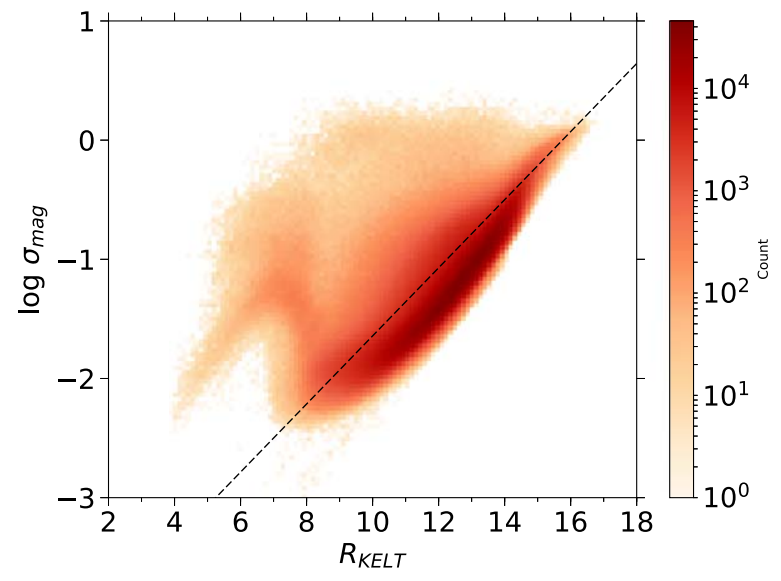

Figure 4. Two-dimensional histogram of the $R_{\mathrm{KELT}}$ and standard deviation of magnitudes of the eastern and western KELT light curves in the color cut sample. Objects with either an eastern or western light curve above the dashed line were selected as candidate variables and kept for further study. The increase in standard deviations at brighter magnitudes is due to saturation effects.

features are used at each decision node. RF has proven effective in classifying variable stars (Dubath et al. 2011; Richards et al. 2011; Hernitschek et al. 2016; Pashchenko et al. 2018) and in some cases has been shown to outperform other machine-learning classifiers such as support vector machines (Yuan et al. 2017).

The RF classifier requires features for each star that ideally characterize the object. The classifier also needs a training set of stars with features and known labels (e.g., Mira-like or not). Once the RF classifier is trained, it can be used to classify a set of stars with unknown classifications. We used SCIKIT-LEARN (Pedregosa et al. 2012), an open-source machine-learning library for PYTHON, to build our RF classifier. For each star passing the cuts in Section 3.1, we determined 16 features. Of these, 5 are determined from the combined light curve data, 3 are from a period search, and 8 are photometric properties from KELT and 2MASS. All features are listed in Table 1 along with the source of the feature (light curve, periodogram, photometry) and the rank of the feature's importance in the RF classification as determined by SCIKIT-LEARN. These features are discussed in the following subsections.

\subsubsection{Features from Photometry}

The features from KELT and 2MASS photometry are $R_{\mathrm{KELT}}$, $J, H, K_{s},\left(R_{\mathrm{KELT}}-J\right),(J-H),\left(J-K_{s}\right)$, and $\left(H-K_{s}\right)$. From Section 3.1 we found that most Miras and other red variables have $\left(H-K_{s}\right)>0.15$, and we found that Miras in our VSX sample are shifted toward redder $H-K_{s}$ colors compared to non-Mira red variables. We therefore retained this color as an RF feature and kept the remaining colors and magnitudes as RF features for comparison. Consulting Table 1, though, we see that, aside from $H-K_{s}$, these photometric features have the least importance in classifying our Mira-like objects.

\subsubsection{Features from the Light Curve}

From each combined light curve we determined the standard deviation of magnitudes $\sigma_{\text {mag }}$, 10th-90th percentile amplitude $A_{10,90}, 1$ st-99th percentile amplitude $A_{1,99}$, Welch-Stetson $L$ (Stetson 1996), and the Alarm variability statistic (Tamuz et al. 2006). Both Welch-Stetson $L$ and Alarm statistics are measures
Table 1

Features for the Random Forest Classifier

\begin{tabular}{llc}
\hline \hline Feature & Source & Rank \\
\hline$\sigma_{\text {mag }}$ & Light curve & 1 \\
$A_{10,90}$ & Light curve & 2 \\
$A_{1,99}$ & Light curve & 3 \\
Welch-Stetson $L$ & Light curve & 4 \\
LS power & Periodogram & 5 \\
Alarm & Light curve & 6 \\
$H-K_{s}$ & Photometry & 7 \\
LS Period & Periodogram & 8 \\
LS S/N & Periodogram & 9 \\
$R_{\text {KELT }}$ & Photometry & 10 \\
$R_{\text {KELT }}-J$ & Photometry & 11 \\
$J-H$ & Photometry & 12 \\
$K_{S}$ & Photometry & 13 \\
$J-K_{S}$ & Photometry & 14 \\
$J$ & Photometry & 15 \\
$H$ & Photometry & 16 \\
\hline
\end{tabular}

of coherent variability and make use of magnitude residuals relative to a weighted mean light curve magnitude. The implementations for calculating the Welch-Stetson $L$ and Alarm statistics were both provided by VARTOOLS, an opensource set of programs for analyzing time-series data (Hartman $\&$ Bakos 2016). The Welch-Stetson $L$ statistic searches for variability by measuring the time-dependent correlation of residuals of magnitude pairs. The time span for magnitude pairs was set to 10 days. The Alarm statistic was originally designed for detecting eclipsing binary star light curves and measures the correlation of adjacent residuals with the same magnitude sign. A large Alarm statistic value indicates that there are a large number of sequential residuals with the same sign that should correlate to coherent variability in the light curve.

We have not applied a hard amplitude cutoff to our data set to separate Miras from SRs for multiple reasons. As discussed in Section 1, although SRs typically have smaller amplitudes than Miras, the conventional amplitude limit is arbitrary, and high-amplitude SRs may have similar characteristics to Miras. Also, using an amplitude criterion with KELT data has problems. First, there exists no conventional amplitude cutoff for identifying Miras using the KELT bandpass. Second, yearly gaps in the observed data may result in incomplete coverage across a pulsation cycle of a star that hinders our ability to accurately measure amplitudes of some variables. Because of these difficulties, we find it hard to disentangle Miras from SRs in our catalog, and we note that we have included in our catalog SRs with similar light curve characteristics to Miras.

\subsubsection{Features from the Periodogram}

We also calculated the Lomb-Scargle (LS) periodogram (Lomb 1976; Scargle 1982; Zechmeister \& Kürster 2009) of the KELT light curves using ASTROPY (Astropy Collaboration et al. 2013, 2018). We tested the use of other period-finding algorithms, such as phase dispersion minimization (Stellingwerf 1978) and Analysis of Variance period search (SchwarzenbergCzerny 1989; Devor 2005), but found LS to be most effective. From the LS periodogram we used the following features for our RF classifier: the combined light curve LS period, the maximum normalized LS power, and a measure of the periodogram $\mathrm{S} / \mathrm{N}$. 
We searched for periods between 50 days and half the baseline of the KELT object under analysis using a frequency spacing of $\Delta f=10^{-5}$ days $^{-1}$ and used the maximum LS power to select periods. This period search was applied to the eastern, western, and combined light curves separately. We adopted the period from the combined data as the correct period but verified that the period was found in eastern and western data. We discuss cases in which the eastern and western periods disagreed, requiring visual inspection later in Section 4.1.

The LS power is a dimensionless value that measures how well a sinusoidal model of a particular frequency, amplitude, and phase fits the light curve data. It is normalized such that a sinusoidal model that perfectly fits the data would result in a power of 1 and a constant nonvarying model would result in a power of 0 . The variables we are interested in have quasiregular periodic signals; therefore, their periodograms should have higher LS powers compared to less regular variable stars. There are distortions and power leakages in the power spectra of both Mira-like and non-Mira stars. Irregular cadence and gaps in the observations cause aliases in our data; variability departing from a perfect sinusoid can also cause harmonics of the true frequency to appear in the spectrum, and multiperiodicity, changing periods, and variable amplitudes can all increase the powers of spurious frequencies. We therefore determined the quality of the periodogram results by calculating an $\mathrm{S} / \mathrm{N}$ for the max power similar to that used by Benko \& Csubry (2007) and Hartman \& Bakos (2016). This is defined as $\mathrm{S} / \mathrm{N}_{\mathrm{LS}}=\left(\mathrm{LS}_{\text {peak }}-\langle\mathrm{LS}\rangle\right) / \mathrm{rms}_{\mathrm{LS}}$, where $\mathrm{LS}_{\text {peak }}$ is the maximum periodogram power, $\langle\mathrm{LS}\rangle$ is the mean of the periodogram, and $\mathrm{rms}_{\mathrm{LS}}$ is the rms of the periodogram powers. This value compares the peak periodogram power to the background periodogram signal. Periodograms with low power leakage will have sharply defined peaks at the pulsation frequency (and at aliases and harmonics) and low power values at all other frequencies. This results in a large $\mathrm{S} / \mathrm{N}$ value when compared to periodograms with higher power leakage that exhibit higher power values across multiple spurious frequencies. Mira-like variables, with their more regular periodic behavior, will exhibit periodograms with larger $\mathrm{S} / \mathrm{N}$ values than irregular variables. We should note that the noise of a periodogram is not normally distributed; therefore, the $\mathrm{S} / \mathrm{N}$ value does not follow a normal distribution. The $\mathrm{S} / \mathrm{N}$ value is also sensitive to light curve baselines, as shorter-baseline observations cause broader periodogram peaks, which increases the value of $\langle\mathrm{LS}\rangle$ and $\mathrm{rms}_{\mathrm{LS}}$. For each object we also calculated upper and lower error estimates on our periods using the FWHM of the periodogram peaks. We attempted to determine errors using Equation (52) of VanderPlas (2018) but found that this method underestimates errors, while the chosen method of using the periodogram peak FWHM overestimates errors.

\subsubsection{Training and Running Random Forest}

After determining features for all objects passing the cuts discussed in Section 3.1, we trained the RF classifier on a sample of VSX objects. We cross-matched our KELT catalog to VSX stars classified as Mira, SR, irregular, or miscellaneous (these are typically unclassified irregulars according to VSX) using a 6 " search radius. We then retained only the VSX stars that passed the color, rms, and amplitude cuts of Section 3.1. We labeled the VSX Miras as Mira-like and inspected a sample of 299 high-amplitude $\left(\Delta R_{\mathrm{KELT}}>1.0\right) \mathrm{VSX} \mathrm{SR}$, irregular, and miscellaneous stars. Of these, we reclassified 172 as Mira-like, as their amplitudes and near-regular periodicity were similar to lower-amplitude Mira light curves. We labeled the remaining $\mathrm{SR}$, irregular, and miscellaneous objects as non-Miras. This resulted in a training set of 13,175 objects, 2453 (19\%) of which were classified as Mira-like.

We used SCIKIT-LEARN to train an RF classifier of 500 decision trees, each with a maximum depth of 20 , on the training set. The classifier returns probabilities of each object in the training set to be classified as Mira-like. To check for misclassified VSX objects in our training set, we used a 10-fold cross-validation and found that a classification score threshold of 0.5 resulted in a sample with $92 \%$ purity and $88 \%$ completeness. We adopted this score to create a preliminary sample of 255 false negatives (VSX Miras misclassified in the cross-validation set) and 182 false positives (VSX non-Miras misclassified as Miras in the cross-validation set). We visually inspected the light curves of these objects and reclassified 201 VSX objects. These new classifications were then introduced into our training set, and the RF was retrained. A 10-fold crossvalidation was then used to determine that a classification score of 0.5 returned an improved sample of Mira-like objects with 94\% purity and $91 \%$ completeness. We applied the trained RF classifier on KELT light curves passing the cuts described in Section 3.1. Objects with classification scores greater than 0.5 were classified as Mira-like and selected for further study. The magnitudes for this classified sample were systematically fainter than the training set, as the training set mostly included well-known, previously classified variables. Magnitudes do not have a direct affect on the RF classifier, as they are a feature with low significance (see Section 3.2.1), but faint magnitudes can affect the values of other features. At fainter magnitudes light curves can exhibit more noise, which can decrease variability statistics such as Welch-Stetson $L$ and Alarm. Fainter-magnitude stars may also be more susceptible to blending from nonvariables. This can decrease their light curve amplitudes. We are unable to correct for these in the KELT data, and we note it as a limitation.

\section{Results}

\subsection{Results from Random Forest}

Our RF classifier returned 4491 Mira-like variables. Light curves of these Mira-like stars were selected for visual inspection if the selected frequency of the eastern, western, or combined periodograms was located at the edge of the frequency grid used (implying that the true period is outside the frequency grid); if either the eastern, western, or combined periods were greater than 1000 days; or if the eastern, western, or combined frequencies differed by more than 0.0005 day $^{-1}$. These criteria resulted in an inspection of the light curves of 525 of the 4491 Mira-like objects. Of these, 130 stars were removed from the catalog because of either ill-defined periods caused by irregularities in their light curve or poor time baseline coverage, 62 stars had their periods redetermined by selecting periodogram peaks that resulted in cleaner phasefolded plots, and 333 stars required no change in period because their combined light curve periods were deemed correct.

We also attempted to identify and correct for issues due to blending by removing false positives due to contamination from nearby variables. Nonvariable objects suffering from blending with a nearby variable have light curve periods nearly 
identical to the variable's period. We identified contaminated light curves by searching for groups of neighboring Mira-like objects in our catalog. Mira-like stars that were within $3^{\prime}$ of each other and had a period difference of less than $5 \%$ were selected as a group. The object in each group with the brightest $J$ magnitude was kept, and the remaining objects were removed from the catalog. This resulted in the removal of 229 objects from the Mira-like catalog.

After visually inspecting the low-quality set, correcting for blending, and removing the above-mentioned stars, we produced a final catalog of 4132 Mira-like objects. Henceforth, we refer to this catalog of 4132 objects as the KELT Mira-like catalog. These are listed in Table 2. A sample of 14 light curves representative of our catalog are shown in Figure 1.

\subsection{Content and Completeness of Our Catalog}

We compared our KELT Mira-like catalog to the catalog of Miras and SRs in VSX, as well as several other catalogs. One of the difficulties in doing this is in identifying which stars in the other catalogs we would expect to be able to detect with KELT. That is partly due to the different magnitude regimes probed by the different projects. The VSX catalog lists the magnitude at maximum amplitude for each object, but these magnitudes are not consistently assembled from $V$-band observations but from a variety of passbands. This hinders determining whether a VSX object is within the KELT magnitude range. To obtain consistent $V$-band magnitudes for the VSX sample, we matched the 15,073 VSX objects to the UCAC survey (Zacharias et al. 2013) using a 6" search radius and selected the nearest match. Not all VSX objects had UCAC $V$ magnitudes, but of the VSX objects that did, we found that approximately half (52\%) were within the KELT magnitude range $(8<V<13)$.

Of the 78,550 Miras and SRs in the VSX catalog with 2MASS-matched colors, 15,073 overlap with the KELT footprint, and we find 7951 (52.8\%) of those in the KELT catalog. This fraction is similar to the fraction of VSX objects in the KELT magnitude range using UCAC $V$ magnitudes, suggesting that we are recovering most VSX objects within the KELT magnitude range.

For a further test of our cross-matching procedure, we inspected a sample of VSX objects that failed to match to KELT but had UCAC $V$ magnitudes within the KELT magnitude range. We found that nearly all failed matches were due to blending of the VSX variable with a nearby bright source in the KELT images. The remaining failed matches were due to the VSX object lying near the edge of a KELT field. The PSFs of these objects are distorted in KELT, causing errors in their astrometric positions in the KELT catalog.

We also found a small sample (180 objects) of dim VSX objects that match to a KELT object but have UCAC $V$ magnitudes outside the KELT magnitude range. These stars are faint in $V$ but are red enough to be detected by KELT's broad $R$ filter. It is also possible that a portion may be incorrect matches either between VSX and KELT or between VSX and UCAC.

The 7951 VSX Miras and SRs that match to stars in the KELT catalog can be broken up into 2228 Miras and 5723 SRs. Our KELT Mira-like catalog recovered 2072 (93\%) of these VSX Miras and only $623(11 \%)$ of these VSX SRs. This suggests that our RF classifier performs well in identifying the Miras that exist in the KELT catalog. The RF classifier does not identify a large portion of the VSX SRs, though, but this should not be alarming, as our study focuses on high-amplitude, regularly periodic stars. Therefore, it is expected that we exclude the majority of SRs that exhibit small-amplitude or irregular pulsations.

In addition to the VSX Miras and SRs, our Mira-like catalog also includes other classification types from VSX. We find 54 LPVs (LPVs of unspecified type), 480 irregulars, 226 MISC stars (miscellaneous variable stars that failed to be classified by automatic analysis), and 59 VAR stars (variable stars of unspecified type) as identified by the VSX catalog. We also find 24 stars of various VSX classified types, with each type having fewer than four matches in our catalog, e.g., our catalog includes three RV Tauri stars, three UX Orionis stars, etc. The final matches between our KELT Mira-like catalog and VSX indicate that $58 \%$ of the members in our catalog are classified as Miras, $18 \%$ as SRs, and $24 \%$ as other variability types, but an unknown fraction of these assorted VSX types (e.g., LPV, MISC) could be reclassified as either Miras or SRs.

For a better determination of the fraction of Miras and SRs in our KELT Mira-like catalog, we used the Woźniak et al. (2004b) catalog of red variables in NSVS (hereafter referred to as W04). The W04 catalog contains 8678 red variable objects, 7157 of which were within the KELT fields. We matched the W04 catalog to the KELT catalog using a $20^{\prime \prime}$ radius (the NSVS pixel size is 14!"4) and found KELT light curves for 5437 objects. W04 classifies these objects into 416 carbon stars (C), 1092 Miras (M), and 3929 SRs or irregulars (SR+L). Our KELT Mira-like catalog recovered 1836 W04 objects: 179 are class C, 1055 are class M, and 602 are class SR+L. Most W04 objects that our KELT Mira-like catalog failed to recover are the low-amplitude SRs. We also found that most W04 class C objects showed irregular periodic behavior, which is expected for carbon stars owing to their asymmetric mass loss. This irregularity is the likely cause of the RF classifier not recovering the class $\mathrm{C}$ objects alongside the more regularly periodic Miras. Of the W04 objects, approximately 67\% are classified as class $\mathrm{C}$ or $\mathrm{M}$, and $33 \%$ are class $\mathrm{SR}+\mathrm{L}$. From this we assume that $\sim 70 \%$ of stars in our KELT Mira-like catalog are Miras and $\sim 30 \%$ are SRs.

The Vogt et al. (2016) catalog of Miras in ASAS (hereafter referred to as V16) contains 2875 objects, 918 of which were within the KELT fields. These were matched to the KELT catalog using a $20^{\prime \prime}$ radius (the ASAS pixel size is $15^{\prime \prime}$ ). We found light curves for 723 objects and successfully recovered 554 of the V16 Miras in our KELT Mira-like catalog. Every V16 Mira was found to have an VSX match. Those objects in either the W04 or V16 catalog with no KELT light curves are either outside the KELT magnitude limit, near the edges of the KELT fields, or blended with nearby objects.

We also compared our results to the Oelkers et al. (2018) catalog of 52,741 variable KELT objects. We have used an improved scheme of cross-matching KELT to 2MASS (see Section 2.2) compared to Oelkers et al. (2018), as they matched KELT to 2MASS by selecting the nearest positional match without incorporating color or magnitude information. We cross-matched our KELT Mira-like catalog to Oelkers et al. (2018) using a search radius of $50^{\prime \prime}$. This radius was chosen to account for cases where the 2MASS identification, and therefore 2MASS coordinates, of KELT objects differed between our catalog and theirs. Out of our 4132 KELT Miralike objects, 2848 cross-matched to the Oelkers et al. (2018) catalog. Of these, 345 had periods listed by Oelkers et al. (2018), 


\begin{tabular}{|c|c|c|c|c|c|c|c|c|c|c|c|c|c|}
\hline Eastern KELT I.D. & Western KELT I.D. & 2MASS I.D. & $\begin{array}{l}\text { R.A. } \\
\text { (J2000) }\end{array}$ & $\begin{array}{l}\text { Decl. } \\
\text { (J2000) }\end{array}$ & $\begin{array}{c}l \\
(\mathrm{deg})\end{array}$ & $\begin{array}{c}b \\
(\mathrm{deg})\end{array}$ & $\begin{array}{l}R_{\mathrm{KELT}} \\
(\mathrm{mag})\end{array}$ & $\begin{array}{c}J \\
(\mathrm{mag})\end{array}$ & $\begin{array}{c}H \\
\text { (mag) }\end{array}$ & $\begin{array}{c}K_{s} \\
(\mathrm{mag})\end{array}$ & $\begin{array}{c}\text { Period } \\
\text { (days) }\end{array}$ & $\mathrm{S} / \mathrm{N}_{\mathrm{LS}}$ & Mismatch Flag \\
\hline KELT N01 lc 012200 V01 east & KELT N01 lc 039679 V01 west & 2MASS J23573235+3202204 & 23:57:32.36 & $+32: 02: 20.48$ & 109.820255 & -29.456737 & 11.05 & 06.08 & 05.30 & 04.88 & $227_{-7}^{+7}$ & 06.19 & 0 \\
\hline KELT N01 lc 012821 V01 east & KELT N01 lc 046147 V01 west & 2MASS J00594645+2756445 & $00: 59: 46.46$ & $+27: 56: 44.58$ & 125.176497 & -34.889698 & 10.19 & 07.18 & 06.38 & 05.95 & $187_{-4}^{+8}$ & 06.05 & 0 \\
\hline KELT N01 lc 029631 V01 east & KELT N01 lc 048055 V01 west & 2MASS J23535704+3310562 & 23:53:57.05 & $+33: 10: 56.27$ & 109.302805 & -28.163464 & 12.18 & 08.18 & 07.25 & 06.92 & $581_{-47}^{+72}$ & 05.26 & 0 \\
\hline KELT N01 lc 033544 V01 east & KELT N01 lc 009353 V01 west & 2MASS J23514408+2945435 & 23:51:44.09 & $+29: 45: 43.56$ & 107.755008 & -31.344054 & 09.07 & 04.62 & 03.75 & 03.14 & $322_{-17}^{+29}$ & 05.70 & 0 \\
\hline KELT N01 lc 033596 V01 east & KELT N01 lc 023249 V01 west & 2MASS J00234610+3830077 & $00: 23: 46.11$ & $+38: 30: 07.73$ & 117.007674 & -24.051108 & 10.88 & 08.92 & 08.13 & 07.93 & $412_{-28}^{+35}$ & 06.04 & 1 \\
\hline KELT N01 lc 041955 V01 east & KELT N01 lc 030143 V01 west & 2MASS J00500630+3539098 & 00:50:06.31 & $+35: 39: 09.89$ & 122.627460 & -27.218213 & 09.36 & 05.48 & 04.69 & 04.27 & $260_{-10}^{+11}$ & 05.94 & 0 \\
\hline KELT N01 lc 043787 V01 east & KELT N01 lc 046756 V01 west & 2MASS J00141091+2901203 & 00:14:10.92 & $+29: 01: 20.33$ & 113.200899 & -33.144494 & 10.39 & 05.69 & 04.84 & 04.44 & $238_{-11}^{+8}$ & 05.77 & 0 \\
\hline KELT N01 lc 046801 V01 east & nan & 2MASS J00471892+3241083 & 00:47:18.93 & $+32: 41: 08.38$ & 121.928508 & -30.178056 & 10.66 & 03.05 & 02.23 & 01.76 & $429_{-34}^{+30}$ & 05.58 & 0 \\
\hline KELT N01 lc 047788 V01 east & nan & 2MASS J00512328+3422363 & $00: 51: 23.29$ & $+34: 22: 36.32$ & 122.920217 & -28.494993 & 10.89 & 04.88 & 03.84 & 03.25 & $329_{-18}^{+21}$ & 05.16 & 0 \\
\hline KELT N01 lc 051102 V01 east & nan & 2MASS J00030086+3038233 & 00:03:00.86 & $+30: 38: 23.34$ & 110.768735 & -31.085668 & 13.41 & 10.90 & 09.71 & 08.90 & $306_{-13}^{+16}$ & 05.74 & 0 \\
\hline KELT N01 lc 051823 V01 east & KELT N01 lc 045548 V01 west & 2MASS J23491137+2638088 & $23: 49: 11.37$ & $+26: 38: 08.89$ & 106.078971 & -34.190596 & 09.22 & 05.33 & 04.20 & 03.80 & $230_{-6}^{+8}$ & 06.37 & 0 \\
\hline KELT N01 lc 052269 V01 east & KELT N01 lc 017514 V01 west & 2MASS J23461773+3024468 & $23: 46: 17.74$ & $+30: 24: 46.88$ & 106.650150 & -30.390832 & 11.08 & 06.04 & 05.14 & 04.75 & $129_{-2}^{+3}$ & 06.30 & 0 \\
\hline KELT N01 lc 053684 V01 east & KELT N01 lc 058300 V01 west & 2MASS J00322275+2601459 & $00: 32: 22.76$ & $+26: 01: 45.94$ & 117.594322 & -36.644720 & 07.80 & 03.42 & 02.54 & 02.13 & $310_{-14}^{+15}$ & 07.05 & 0 \\
\hline KELT N01 lc 053981 V01 east & KELT N01 lc 048462 V01 west & 2MASS J00222314+2659458 & $00: 22: 23.15$ & $+26: 59: 45.83$ & 114.985647 & -35.427023 & 08.69 & 04.68 & 03.92 & 03.50 & $279_{-13}^{+14}$ & 05.86 & 0 \\
\hline KELT N01 lc 054306 V01 east & KELT N01 lc 049456 V01 west & 2MASS J00482082+2703259 & $00: 48: 20.83$ & $+27: 03: 25.98$ & 122.083382 & -35.809439 & 10.99 & 05.13 & 04.17 & 03.81 & $344_{-32}^{+15}$ & 05.18 & 0 \\
\hline KELT N01 lc 056383 V01 east & KELT N01 lc 045232 V01 west & 2MASS J23430657+3528452 & $23: 43: 06.58$ & $+35: 28: 45.21$ & 107.583772 & -25.347799 & 10.89 & 04.92 & 03.91 & 03.24 & $377_{-11}^{+31}$ & 07.09 & 0 \\
\hline KELT N01 lc 058729 V01 east & KELT N01 lc 058434 V01 west & 2MASS J00000657+2553112 & 00:00:06.57 & $+25: 53: 11.23$ & 108.712957 & -35.564066 & 08.01 & 02.23 & 01.32 & 00.92 & $321_{-14}^{+27}$ & 06.18 & 0 \\
\hline KELT N01 lc 060087 V01 east & KELT N01 lc 058910 V01 west & 2MASS J23505191+4304452 & 23:50:51.92 & $+43: 04: 45.21$ & 111.329781 & -18.423770 & 12.71 & 07.67 & 06.84 & 06.50 & $280_{-12}^{+11}$ & 06.33 & 0 \\
\hline KELT N01 lc 065529 V01 east & KELT N01 lc 059071 V01 west & 2MASS J00042008+4006356 & 00:04:20.08 & $+40: 06: 35.64$ & 113.249082 & -21.874575 & 08.67 & 03.57 & 02.59 & 02.08 & $315_{-16}^{+13}$ & 07.11 & 0 \\
\hline KELT N01 lc 066205 V01 east & KELT N01 lc 049416 V01 west & 2MASS J23572739+2407312 & 23:57:27.39 & $+24: 07: 31.21$ & 107.440420 & -37.117208 & 11.53 & 06.59 & 05.72 & 05.25 & $244_{-6}^{+10}$ & 06.78 & 0 \\
\hline
\end{tabular}

(This table is available in its entirety in machine-readable form.) 
and 30 of these periods differed from ours by more than $1 \%$. We found 1255 KELT objects where the Oelkers et al. (2018) listed 2MASS identification differed from our catalog.

We also matched our KELT Mira-like catalog to the Gaia Data Release 2 (Gaia DR2; Gaia Collaboration et al. 2018) using a $1^{\prime \prime}$ search radius and selecting the nearest positional match, and we found Gaia DR2 matches for all 4132 objects. We do not make use of the astrometric results from Gaia DR2 in our study for several reasons, though. The huge size of Miras, which have variable diameters that are strongly wavelength dependent, results in angular diameters at least twice the size of their parallaxes (Whitelock \& Feast 2000). Miras with thin dust shells have surface features that are dominated by convection and therefore have shifting photocenters (Freytag et al. 2017). The astrometric errors introduced by this are discussed by Chiavassa et al. (2018) for SR variables and will be even larger for Miras. For Mira stars with appreciable dust shells, which are expected to be patchy, the uncertainties will be larger still (Whitelock 2012). The large angular sizes combined with shifting photocenters from asymmetric light distributions across the disk make the parallax measurements for AGBs currently unreliable. The large variation in magnitude and color of Miras also affects the accurate determination of parallaxes. Currently, Gaia DR2 parallaxes are determined assuming constant magnitude and color for each source. This causes further inaccuracies for Mira parallaxes (Mowlavi et al. 2018). It may be possible to derive good parallaxes for Miras and Gaia, but probably only at the end of the mission.

We also matched our KELT Mira-like catalog to the Gaia DR2 Catalog of LPV Stars (Mowlavi et al. 2018; hereafter referred to as M18). The M18 catalog contains 151,761 LPV candidates with period measurements from Gaia DR2, 23,405 of which M18 have classified into Miras. Using a $1^{\prime \prime}$ search radius and selecting the nearest positional match, we matched our KELT Mira-like catalog to the M18 catalog and found matches for 2440 objects.

We are particularly interested in knowing how many objects in our KELT Mira-like catalog are new Mira-like detections. In addition to the VSX and W04 catalogs, we also made use of GCVS (Samus' et al. 2017), the ASAS-SN (Shappee et al. 2014; Jayasinghe et al. 2019) and SIMBAD databases. We crossmatched our catalog to GCVS using a 10 arcsec search radius and found 2242 matches, 2081 of which were classified as Mira or SR. For the ASAS-SN catalog, we also used a 10 arcsec search radius and found 3813 matches, 3364 of which were classified as Mira or SR. We matched our KELT Mira-like catalog to SIMBAD using a 2 arcsec search radius. For cases in which a coordinate search to SIMBAD produced no results, we attempted a SIMBAD match using the 2MASS ID. This returned 3637 matches between our KELT Mira-like catalog and the SIMBAD database, 3110 of which were classified as a Mira, SR, or related type (e.g., AGB or LPV). These catalogs were used to determine the classification status of our Mira-like stars. The classification schemes of the catalogs are not homogeneous, thus we inspected the classification types used across catalogs and included those related to Miras, such as AGB or LPV, as previously detected Mira-like stars. Our KELT Mira-like catalog contains 376 stars not previously classified as a Mira, SR, or a closely related type. Figure 5 shows light curves of four stars not previously classified as a Mira-like variable.

\subsection{Comparing Periods to Other Catalogs}

We compared the periods of variability we found to those of V16, W04, and M18. V16 used a nonparametric algorithm to reanalyze periods of Miras in the ASAS catalog, which required human interaction at certain stages. The KELT and V16 periods are compared in Figure 6(a) and agree very well. The W04 catalog has a short time baseline nearing $1 \mathrm{yr}$ for the longest observations. Therefore, they were unable to obtain precise periods for many of their Miras. There is a sample of Miras with periods given by W04 as 730 days (their maximum period range), which we have redetermined. Figure 6(b) compares the KELT and W04 periods. Figure 6(c) compares the KELT Mira-like and M18 periods. We see that most periods agree well, but there is more scatter, and approximately 160 stars lie along the common aliases and harmonics. Many of these stars exhibit periods near a year or half a year that can demonstrate spurious signals due to systematic noise on annual timescales in the KELT data.

V16 identified three peaks in their period distribution at 215, 275 , and 330 days. When binning the data into 10 day bins, as done in V16, we find similar peaks in our distribution at 195, 285, and 335 days. Our period distribution is shown in Figure 7, and the periods associated with the V16 identified peaks are represented by black markers. However, we find that the combination of systematic noise and aliasing of periods is responsible for the 195 and 335 day peaks, and only the central peak at 285 days is significant.

To further analyze these peaks, we created a new histogram using only the stars in which our periods agreed with those of M18 within $30 \%$. This distribution is shown in Figure 8 . We see that the 195 day peak disappears because of the removal of mismatched periods. We fit both a Gaussian and skewed Gaussian distribution function to this histogram and found negligible differences in the fit quality. The dashed line in Figure 8 represents a skewed Gaussian fit to the histogram, and the $1 \sigma$ and $2 \sigma$ errors are represented by red and orange shading, respectively. These errors were calculated using the square root of the number of bin counts expected from the distribution function. It is apparent that the 335-day peak in this sample does not appear to be significant. Although a Gaussian is not a perfect fit, the distribution of the entire KELT Mira-like catalog is consistent with a Gaussian profile with a mean period of 285 days and standard deviation of 111 days, and the additional peaks at 195 and 335 days claimed by V16 are not confirmed.

We investigated the dependence of our period distribution on Galactic longitude and latitude. We have used only the sample of stars with KELT periods agreeing within $30 \%$ of the M18 catalog to remove the peaks at 215 and 330 days. Figure 9 shows histograms of the period distributions for four longitude bins. Here we have increased the size of the period bins to 20 days to reduce counting errors. The solid blue histogram shows the periods of stars that lie in the range $l>315^{\circ}$ and $l<45^{\circ}$ (toward the Galactic center). The dashed orange histogram shows stars between $45^{\circ}<l<135^{\circ}$. The dotted-dashed green histogram shows stars between $135^{\circ}<l<225^{\circ}$ (toward the Galactic anticenter), and the dotted red histogram shows stars between $225^{\circ}<l<315^{\circ}$. V16 suggests, using arguments from Feast \& Whitelock (2014), that the period distribution reflects the star formation history of the sampled regions, with shorter periods associated with older stars. Previous studies have shown that oxygen-rich (O-rich) Miras are concentrated toward the Galactic center and that carbon-rich (C-rich) Miras have a more uniform distribution across the Galaxy (Blanco 1965; 
(a)
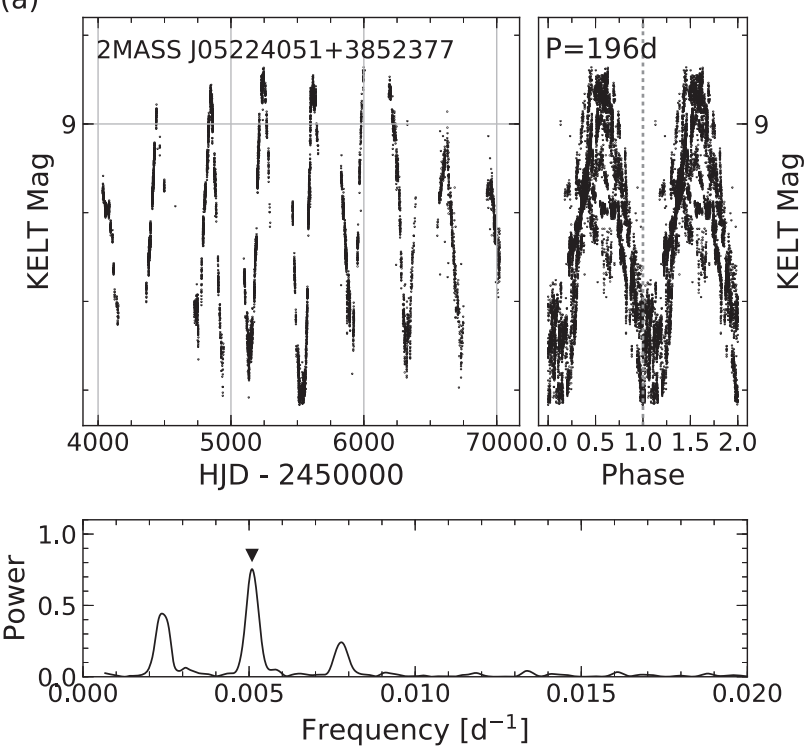

(c)
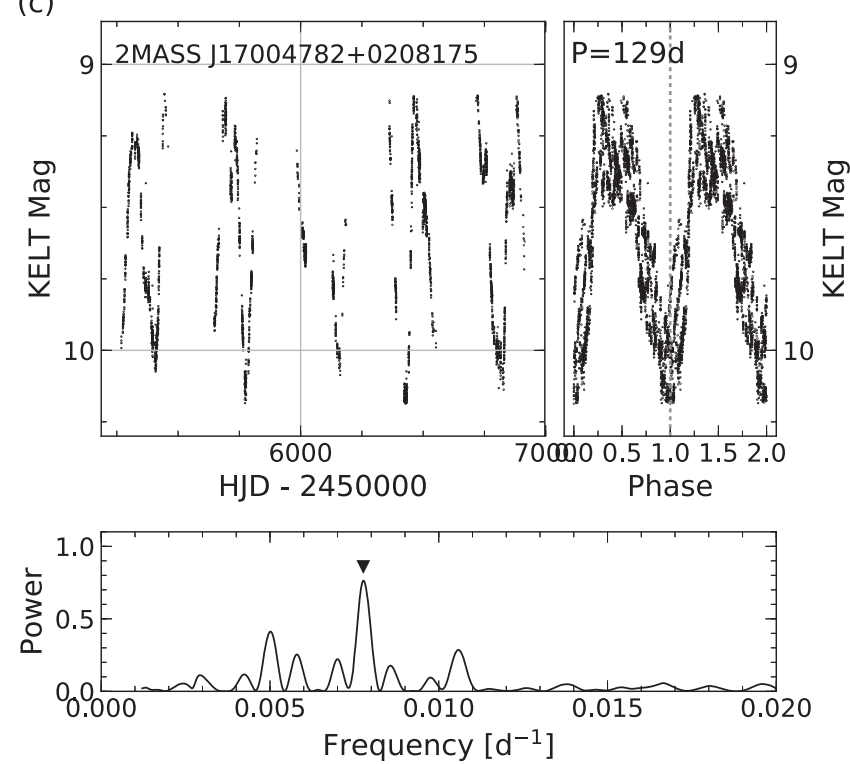

(b)
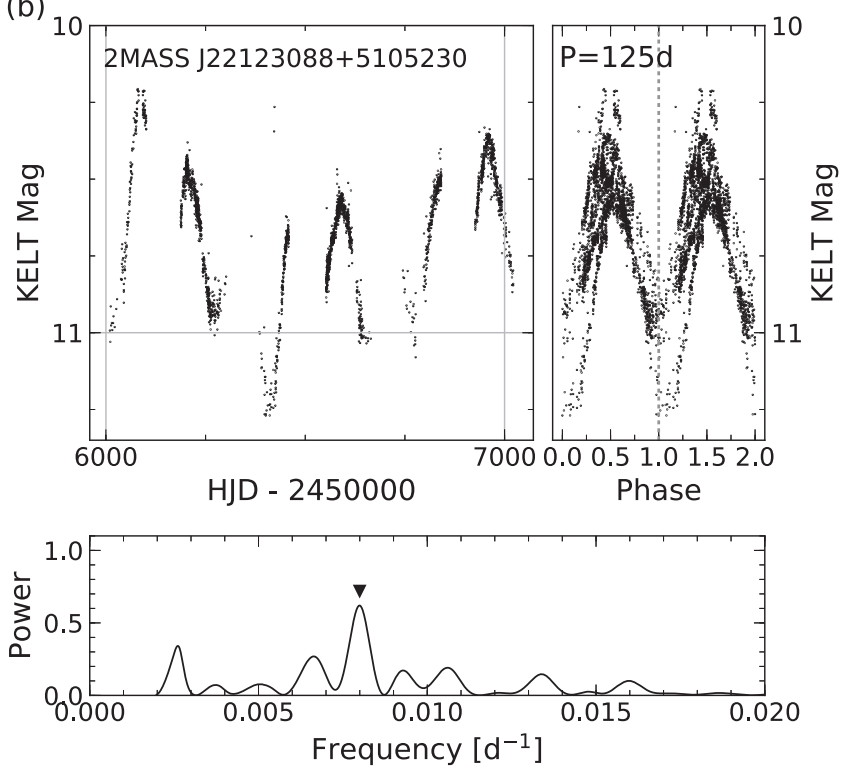

(d)
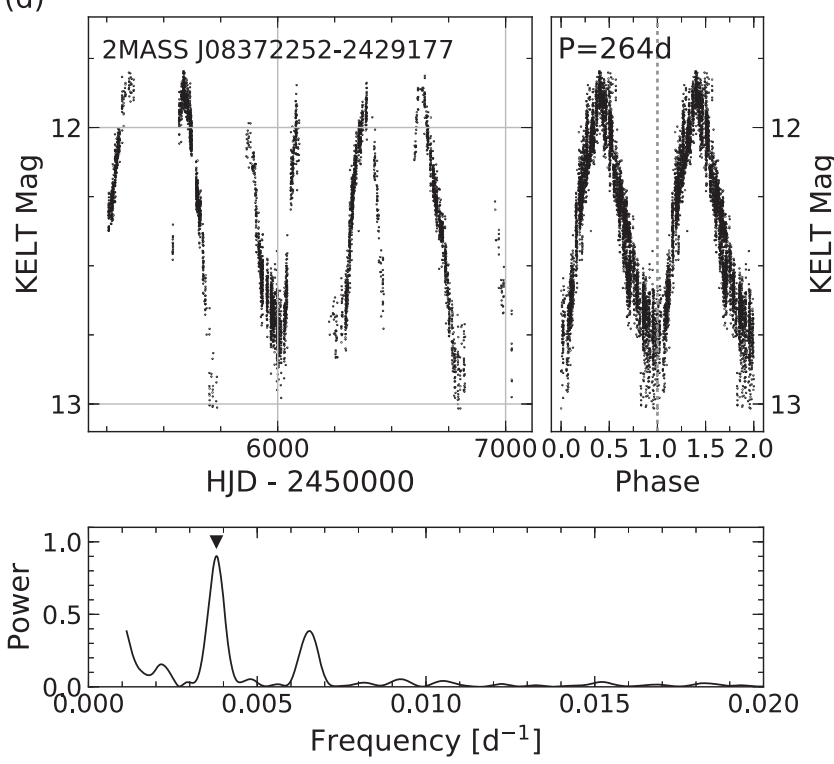

Figure 5. Sample of four KELT light curves from our catalog that have not been previously classified as Mira or SR. The 2MASS identifiers and measured periods are (a) 2MASS J05224051+3852377 with $P=196$ days, (b) 2MASS J22123088+5105230 with $P=125$ days, (c) 2 MASS J17004782 +0208175 with $P=129$ days, and (d) 2MASS J08372252-2429177 with $P=264$ days.

Noguchi et al. 2004; Ishihara et al. 2011 and references therein) and O-rich Miras typically have shorter periods than C-rich Miras. However, we do not confirm a relation between Galactic longitude and KELT periods.

Figure 10 shows period histograms for four latitude bins, still using only KELT periods agreeing within $30 \%$ of M18. The solid blue histogram is for Miras with $|b|<10^{\circ}$, the dashed orange histogram is for Miras between $10^{\circ}<|b|<20^{\circ}$, the dotted-dashed green histogram is for Miras between $20^{\circ}<$ $|b|<30^{\circ}$, and the dotted red histogram is for Miras with $|b|>30^{\circ}$. Generally we find a shift toward shorter periods at higher latitudes. This is consistent with the expectation that longer-period stars have higher-mass progenitors and are therefore more concentrated in the Galactic plane. A similar effect can be seen in Figures 16 and 17 from Whitelock et al. (1994).

\section{Determining Carbon- and Oxygen-rich Types}

Mira variables fall into three subclasses depending on the abundances of oxygen versus carbon in their atmospheres. Miras with similar oxygen and carbon abundances are referred to as S-type, and Miras with an imbalance between oxygen and carbon are referred to as either oxygen-rich (O-rich) or carbonrich (C-rich). C-rich AGBs are typically redder than O-rich ones owing to the reddening by dust in their atmospheres; thus, they typically have redder $J-K_{s}$ colors (Feast et al. 1982; Marigo et al. 2003). Distinguishing O-rich from C-rich Miras is useful for distance studies. Compared to O-rich Miras, the PL relation in $K_{s}$ band breaks down over a longer range of periods for C-rich Miras owing to circumstellar reddening caused by their thicker shells (Whitelock 2012). This results in O-rich Miras having a better-defined PL relation. 

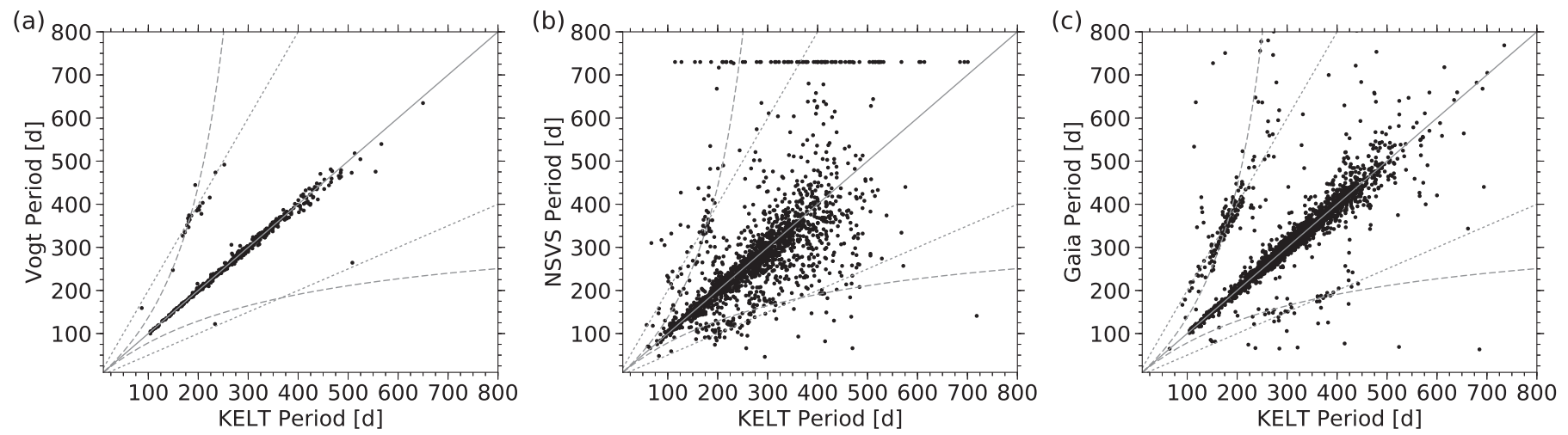

Figure 6. We matched our Mira-like catalog to the catalogs of (a) V16, (b) W04, and (c) M18 to verify the accuracy of our measured periods. Predicted aliases are shown as dashed lines, and period harmonics are shown as straight dotted lines. We find good agreement between our periods and those of V16, with most discrepancies near the harmonics. The periods of W04 appear to be less reliable, likely due to the shorter time baseline of their work. There is also good agreement between our periods and those of M18, but the number of discrepancies along aliases and harmonics is larger.

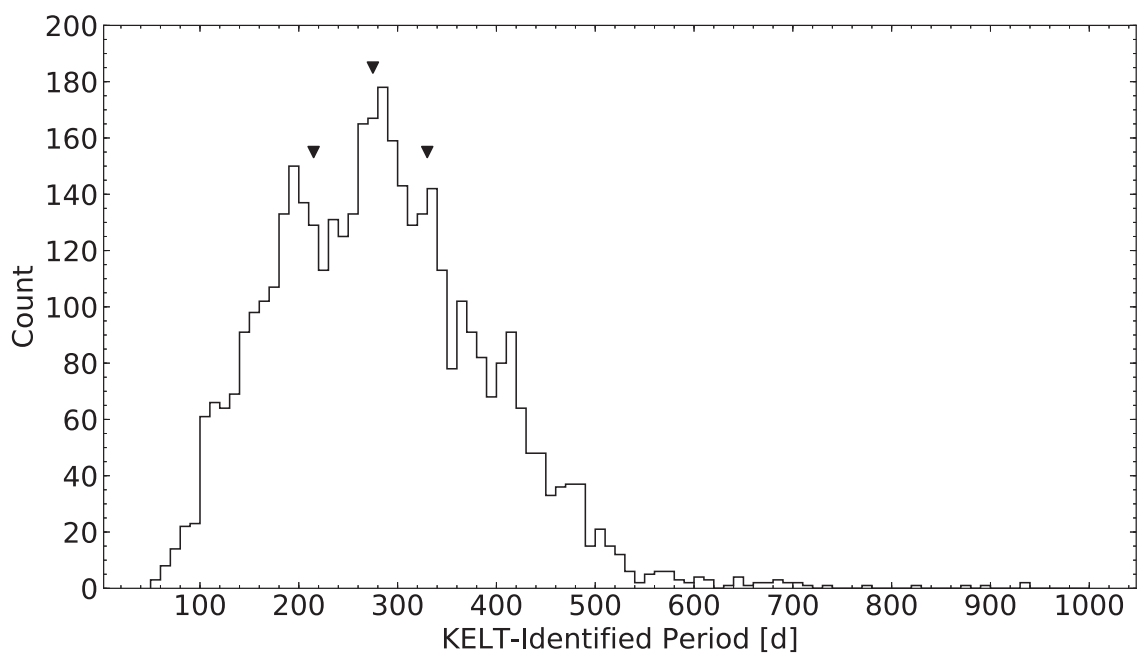

Figure 7. Distribution of periods for our Mira catalog. Period values associated with peaks identified by V16 at 215,275 , and 330 days are marked by triangles. We find that our peak at 195 days is contaminated by mismatched periods, and our 335 day peak is consistent with noise. We therefore cannot confirm the significance of our 195 and 335 day peaks.

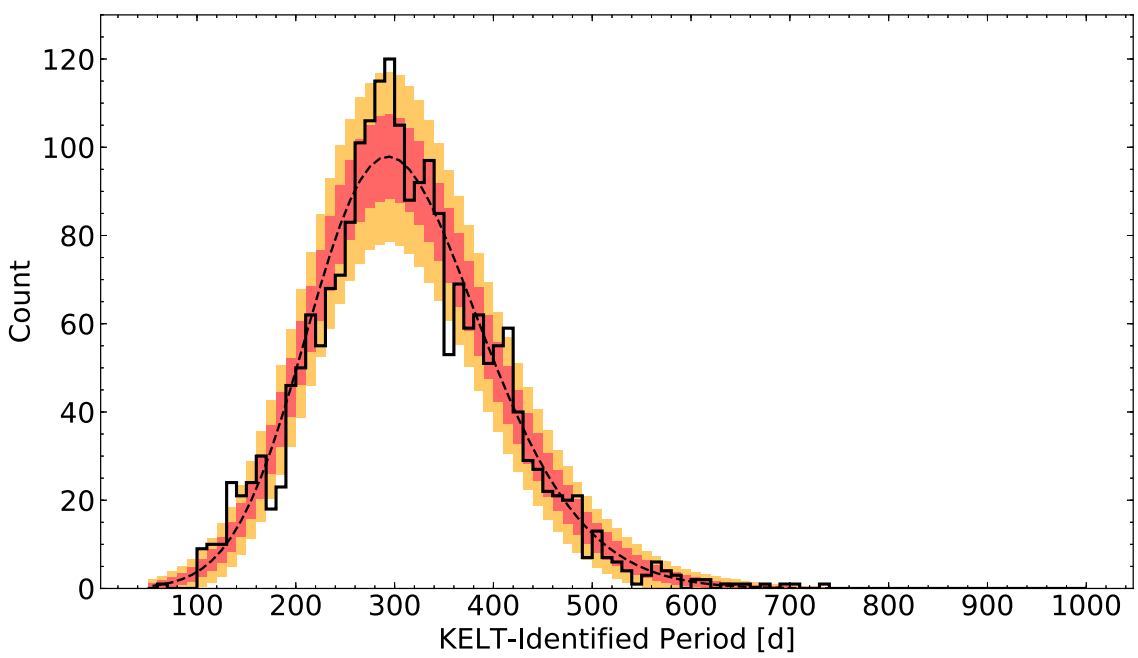

Figure 8. Distribution of periods for stars in which our periods and M18 periods agree within $30 \%$. The dashed line represents a skewed Gaussian distribution function that has been fit to the data. The $1 \sigma$ and $2 \sigma$ confidence levels are shown represented by the red and orange areas, respectively. We see that the 195 -day peak disappears because of the removal of mismatched periods, and the peak near 335 days is consistent with noise. 


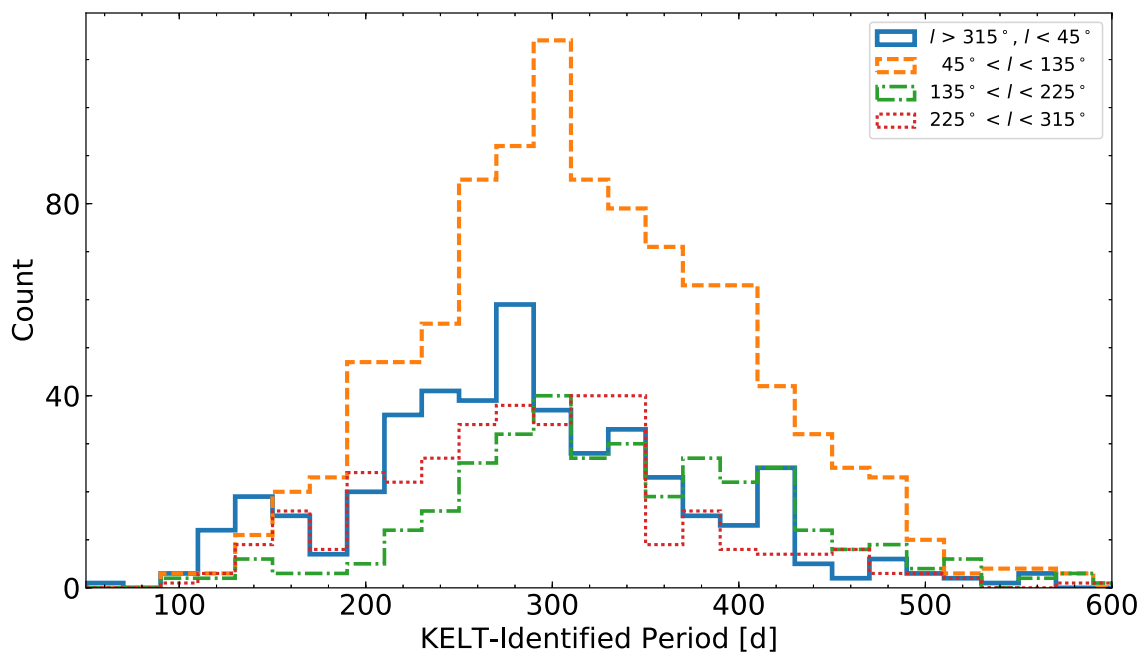

Figure 9. Distribution of periods for stars in which periods agree within 30\% of M18 for four Galactic longitude bins. The solid blue histogram is for Miras between $l>315^{\circ}$ and $l<45^{\circ}$, the dashed orange histogram is for Miras between $45^{\circ}<l<135^{\circ}$, the dotted-dashed green histogram is for Miras between $135^{\circ}<l<225^{\circ}$, and the dotted red histogram is for Miras between $225^{\circ}<l<315^{\circ}$.

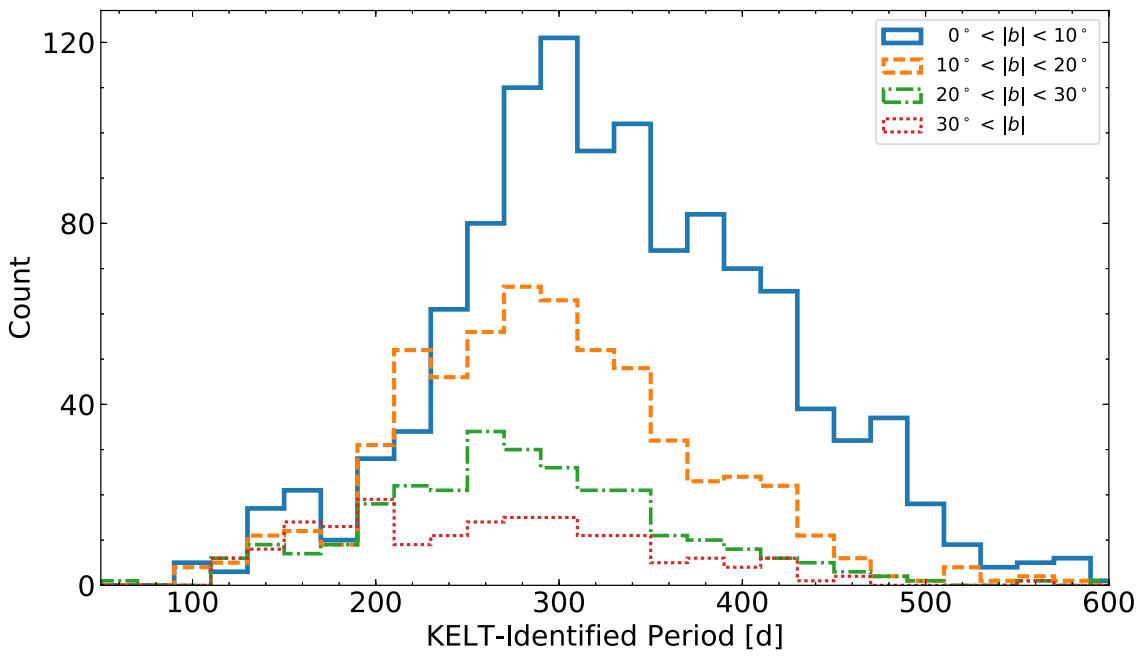

Figure 10. Distribution of periods for stars in which periods agree within $30 \%$ of M18 for four absolute Galactic latitude bins. The solid blue histogram is for Miras with $|b|<10^{\circ}$, the dashed orange histogram is for Miras between $10^{\circ}<|b|<20^{\circ}$, the dotted-dashed green histogram is for Miras between $20^{\circ}<|b|<30^{\circ}$, and the dotted red histogram is for Miras with $|b|>30^{\circ}$. We observe a shift toward lower periods at high latitudes.

$J-K_{s}$ colors have commonly been used to distinguish Ofrom C-rich Miras. Cole \& Weinberg (2002) used the criterion of $\left(J-K_{S}\right)>2$ to classify C-rich stars in the Galactic bulge. However, these results have been questioned, and resulting studies suggest that $J-K_{s}$ colors alone are not accurate in distinguishing between $\mathrm{C}$-rich and O-rich Miras (Ojha et al. 2007; Uttenthaler et al. 2015; Catchpole et al. 2016). Alternative diagnostics in selecting O-rich and C-rich stars from photometry have been explored. Ishihara et al. (2011) and Matsunaga et al. (2017) have used color-color diagrams of $J-K_{s}$ and AKARI colors to select C-rich stars in the Galactic bulge and have shown this to be an improvement over the use of a single 2MASS color. Lebzelter et al. (2018) have had success distinguishing subclasses of AGBs in the Magellanic Clouds using colors from both 2MASS and the Gaia DR2. They were able to distinguish C-rich from O-rich Miras by plotting $K_{s}$ against a combination of 2MASS and Gaia colors known as Wesenheit indices. The Wesenheit index $W_{K_{s}}$ is a reddening-free combination of 2MASS magnitudes defined as

$$
W_{K_{s}}=K_{s}-0.67\left(J-K_{s}\right) \text {. }
$$

This is derived assuming an $R_{V}=3.1$ (Mathis 1990; Dutra et al. 2002; Soszyński et al. 2013). The index $W_{R P}$ is a reddening-free combination of Gaia magnitudes derived by Lebzelter et al. (2018),

$$
W_{R P}=G_{R P}-1.3\left(G_{B P}-G_{R P}\right),
$$

where $G_{B P}$ and $G_{R P}$ are Gaia blue and red apparent magnitudes, respectively. Because the Miras in our catalog are Galactic and their distances are uncertain, we cannot replicate their method of using $K_{s}$ in place of absolute magnitudes. Instead, we investigate the combination of Wesenheit indices $\left(W_{R P}-W_{K_{s}}\right)$ and $\left(J-K_{s}\right)$ colors. The color index $\left(W_{R P}-W_{K_{s}}\right)$ is not a color in the classical sense, but a combination of three colors. In this index, bluer objects are found near $\left(W_{R P}-W_{K_{s}}\right)=0.8$ and redder objects at lower or higher values. The Wesenheit color index 


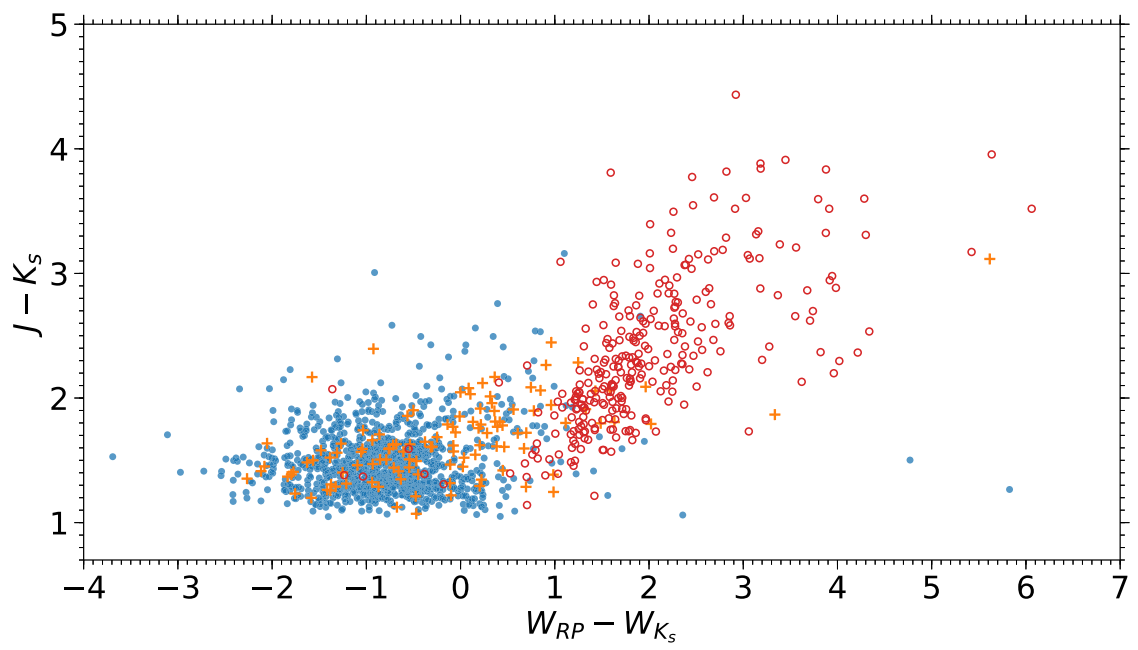

Figure 11. $W_{R P}-W_{K_{S}}$ vs. $J-K_{S}$ for KELT Miras with known spectral types from SIMBAD. O-rich stars are shown as blue filled circles, C-rich as red open circles, and S-type as orange plus signs. Two of the O-rich stars in the lower right with low $J-K_{s}$ and high $W_{R P}-W_{K_{s}}, \mathrm{~V} \mathrm{CVn}$ and RZ Sco, exhibit astrophysical masers.

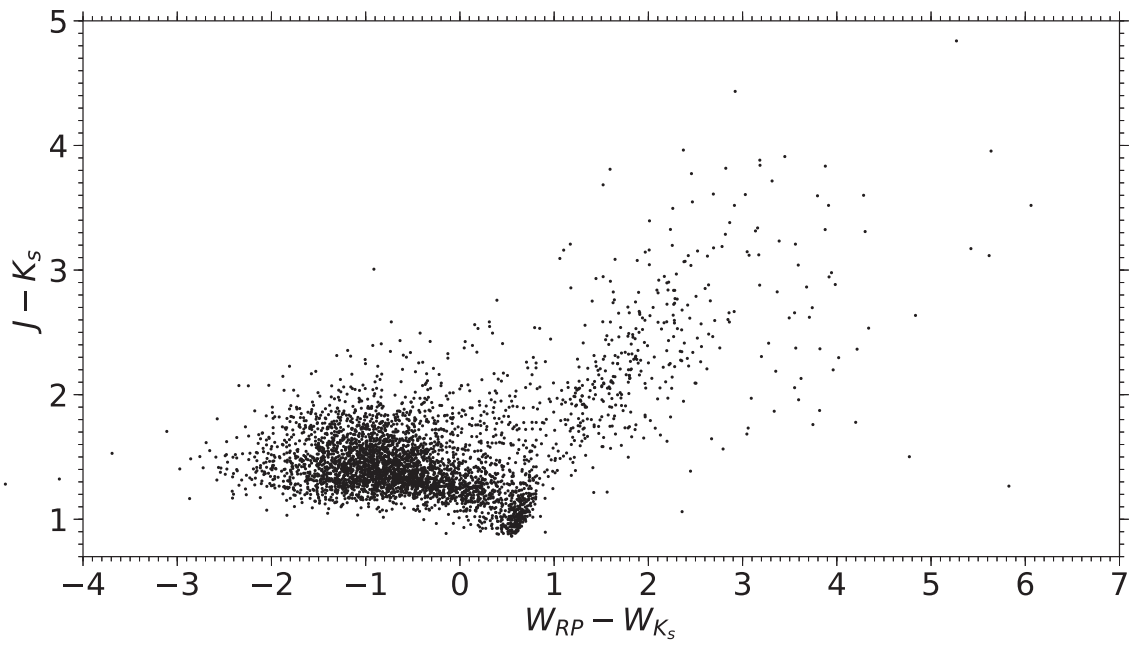

Figure 12. $W_{R P}-W_{K_{s}}$ vs. $J-K_{s}$ for all stars in our KELT Mira-like catalog. Stars with $W_{R P}-W_{K_{s}}<0.8$ are likely O-rich, and those with $W_{R P}-W_{K_{s}}>0.8$ are likely C-rich. The population of stars with low $J-K_{s}$ colors and $W_{R P}-W_{K_{s}}$ colors near 0.5 are likely spurious 2 MASS cross-matches that are flagged in our catalog.

$\left(W_{R P}-W_{K_{s}}\right)$ traces temperature and molecular features in stellar spectra (see Appendix A of Lebzelter et al. 2018).

We first identify the spectral types of our KELT Mira-like catalog by matching to SIMBAD using the method discussed in Section 4.2. Of the 4132 stars in our catalog, we were able to sort 1541 into O-rich, C-rich, or S-type. We then use our Gaia DR2 cross-matched sample to acquire their $G_{R P}$ and $G_{B P}$ magnitudes and determined their Wesenheit indices.

The plot of $\left(W_{R P}-W_{K_{s}}\right)$ versus $\left(J-K_{s}\right)$ for Miras with identified spectral types is shown in Figure 11. Known O-rich stars are shown as blue filled circles, C-rich as red open circles, and S-type as orange plus signs. We can see that $W_{R P}-W_{K_{s}}$ is more effective than $J-K_{s}$ at separating O-rich from C-rich. Even without the use of absolute $K_{s}$ magnitudes, we are generally able to classify stars with $\left(W_{R P}-W_{K_{s}}\right)<0.8$ as O-rich and those with $\left(W_{R P}-W_{K_{\mathrm{s}}}\right)>0.8$ as C-rich. There are three outlying O-rich Miras with low $J-K_{s}$ and high $W_{R P}-W_{K_{s}}$ colors. These three stars are V CVn, SS Oph, and RZ Sco, which respectively lie at $\left(W_{R P}-W_{K_{s}}, J-K_{s}\right)=(5.8,1.3),(4.8,1.5)$, and $(2.4,1.1)$. Of these three, $\mathrm{V} C V n$ has known $\mathrm{OH}, \mathrm{H}_{2} \mathrm{O}$, and $\mathrm{SiO}$ masers, and $\mathrm{RZ}$ Sco has a known $\mathrm{H}_{2} \mathrm{O}$ maser (Kim et al. 2013 and references therein).
The plot of $\left(W_{R P}-W_{K_{s}}\right)$ versus $\left(J-K_{s}\right)$ for our entire KELT Mira-like catalog is shown in Figure 12. The loci of O-rich and C-rich stars still exist, but there also exists a population of approximately 250 stars with $W_{R P}-W_{K_{s}}$ near 0.5 and $J-K_{s}$ near 1.0. The colors of these stars are likely spurious, as most stars in this region were found to have incorrect 2MASS identifications owing to blending. These stars failed to be corrected for blending via the procedure described in Section 4.1 and are flagged in our catalog ("Mismatch Flag" in Table 2).

\section{Summary}

We have compiled a catalog of 4132 high-amplitude, longperiod Mira-like variables using data from the KELT survey. We estimate that our catalog has a completeness of approximately $90 \%$ for finding Miras between $8<V<13$, and that $70 \%$ of our catalog objects are Miras and $30 \%$ are SRs according to the conventional definitions. A total of 376 objects in our catalog have not previously been classified as LPVs such as Miras, SRs, or any other class related to AGB stars.

Comparison to other catalogs reveals very good agreement between both periods, although aliasing is clearly present. The periods for most of our stars lie between 50 and 500 days, with 
a mean period value of 285 days. Additional peaks in the Mira period distribution, as found by V16, are not confirmed. They may not truly exist, but if they do, they are masked by noise and aliasing. Future analysis on stars with longer time baselines could be done to more carefully determine the significance of these peaks. We do find that shorter periods are preferentially found at higher Galactic latitudes, consistent with Whitelock et al. (1994).

Finally, we also a presented a new method to identify O-rich and C-rich stars in our Galaxy, even in the absence of absolute magnitudes, using the Wesenheit color indices discussed by Lebzelter et al. (2018). This is important for future PL studies using Miras. The PL relation of O-rich Miras gives them great potential to rival Cepheids as standard candles in determining distances within the Milky Way and to nearby galaxies. Refined parallax measurements from Gaia will improve the calibration of this PL relation, which will be applicable to future distance studies.

We are grateful to the anonymous referee and the AAS statistics consultant for their comments that significantly improved this manuscript. We would like to thank Shazrene Mohamed for her input on the early stages of this work, and R.A.A. thanks Annika Ewigleben and Alyssa Hanes for constructive criticism of the manuscript. R.A.A. was supported by the NSF grants PHY-0849416 and PHY-1359195. R.A.A. would like to acknowledge support from Lehigh University, specifically financial support through the Doctoral Travel Grant for Global Opportunities and the Summer Research Fellowship from the College of Arts and Sciences, and support from the Department of Physics. R.A.A. would also like to acknowledge support from the IAU in the form of a travel grant. M.V.M. was supported by a Dean's Associate Professor Advancement Fellowship from Lehigh University. P.A.W. acknowledges research funding from the South African NRF. This research has made use of the SIMBAD database and the VizieR catalog access tool, both operated at CDS, Strasbourg, France, and the International Variable Star Index (VSX) database, operated at AAVSO, Cambridge, Massachusetts, USA. We have also made use of ASTROPY, a community-developed core Python package for Astronomy (Astropy Collaboration et al. 2013, 2018); NUMPY (van der Walt et al. 2011); SCIKIT-LEARN (Pedregosa et al. 2012); and MATPLOTLIB, a Python library for publication quality graphics (Hunter 2007). This work has made use of data from the European Space Agency (ESA) mission Gaia (https:// www.cosmos.esa.int/gaia), processed by the Gaia Data Processing and Analysis Consortium (DPAC, https://www.cosmos. esa.int/web/gaia/dpac/consortium). Funding for the DPAC has been provided by national institutions, in particular the institutions participating in the Gaia Multilateral Agreement.

\section{ORCID iDs}

R. Alex Arnold (i) https://orcid.org/0000-0002-3593-0836 M. Virginia McSwain (i) https://orcid.org/0000-00024775-2803

Joshua Pepper (1) https://orcid.org/0000-0002-3827-8417 Patricia A. Whitelock (1) https://orcid.org/0000-00024678-4432

Nina Hernitschek (1) https://orcid.org/0000-0003-1681-0430 David J. James ๑ https://orcid.org/0000-0001-5160-4486

Rudolf B. Kuhn (1) https://orcid.org/0000-0002-4236-9020 Michael B. Lund (1) https://orcid.org/0000-0003-2527-1598
Joseph E. Rodriguez (1) https://orcid.org/0000-00018812-0565

Robert J. Siverd (1) https://orcid.org/0000-0001-5016-3359

Keivan G. Stassun (ํ) https://orcid.org/0000-0002-3481-9052

\section{References}

Alcock, C., Allsman, R. A., Axelrod, T. S., et al. 1993, in Proc. ASP Conf. Ser. 43. Sky Surveys, ed. B. T. Soifer (San Francisco, CA: ASP), 291

Astropy Collaboration, Price-Whelan, A. M., Sipőcz, B. M., et al. 2018, AJ, 156,123

Astropy Collaboration, Robitaille, T. P., Tollerud, E. J., et al. 2013, A\&A, 558, A 33

Bedding, T. R., \& Zijlstra, A. A. 1998, ApJL, 506, L47

Benko, J. M., \& Csubry, Z. 2007, AcA, 57, 73

Blanco, V. M. 1965, in Galactic Structure, Vol. V ed. A. Blaauw \& M. Schmidt (Chicago, IL: Univ. Chicago Press), 241

Breiman, L. 2001, Machine Learning, 45, 5

Cadmus, R. R. 2015, JAVSO, 43, 3

Catchpole, R. M., Whitelock, P. A., Feast, M. W., et al. 2016, MNRAS, 455, 2216

Chiavassa, A., Freytag, B., \& Schultheis, M. 2018, A\&A, 617, L1

Cole, A. A., \& Weinberg, M. D. 2002, ApJL, 574, L43

Devor, J. 2005, ApJ, 628, 411

Dubath, P., Rimoldini, L., Süveges, M., et al. 2011, MNRAS, 414, 2602

Dutra, C. M., Santiago, B. X., \& Bica, E. 2002, A\&A, 381, 219

Feast, M., Whitelock, P., \& Menzies, J. 2002, MNRAS, 329, L7

Feast, M., \& Whitelock, P. A. 2014, in IAU Symp. 298, Setting the Scene for Gaia and LAMOST, ed. S. Feltzing et al. (Cambridge: Cambridge Univ. Press), 40

Feast, M. W., Glass, I. S., Whitelock, P. A., et al. 1989, MNRAS, 241, 375

Feast, M. W., Robertson, B. S. C., Catchpole, R. M., et al. 1982, MNRAS, 201, 439

Freytag, B., Liljegren, S., \& Höfner, S. 2017, A\&A, 600, A137

Gaia Collaboration, Brown, A. G. A., Vallenari, A., et al. 2018, A\&A, 616, A1 Gaia Collaboration, Prusti, T., de Bruijne, J. H. J., et al. 2016, A\&A, 595, A1 Hartman, J. D., \& Bakos, G. Á 2016, A\&C, 17, 1

Hernitschek, N., Schlafly, E. F., Sesar, B., et al. 2016, ApJ, 817, 73

Huang, C. D., Riess, A. G., Hoffmann, S. L., et al. 2018, ApJ, 857, 67

Hughes, S. M. G., \& Wood, P. R. 1990, AJ, 99, 784

Hunter, J. D. 2007, CSE, 9, 90

Ishihara, D., Kaneda, H., Onaka, T., et al. 2011, A\&A, 534, A79

Jayasinghe, T., Stanek, K. Z., Kochanek, C. S., et al. 2019, MNRAS, 485, 961

Kerschbaum, F., \& Hron, J. 1992, A\&A, 263, 97

Kim, J., Cho, S.-H., \& Kim, S. J. 2013, AJ, 145, 22

Lebzelter, T., Mowlavi, N., Marigo, P., et al. 2018, A\&A, 616, L13

Lomb, N. R. 1976, Ap\&SS, 39, 447

Marigo, P., Girardi, L., \& Chiosi, C. 2003, A\&A, 403, 225

Mathis, J. S. 1990, ARA\&A, 28, 37

Matsunaga, N., Menzies, J. W., Feast, M. W., et al. 2017, MNRAS, 469, 4949 Mowlavi, N., Lecoeur-Taïbi, I., Lebzelter, T., et al. 2018, A\&A, 618, A58

Noguchi, K., Aoki, W., \& Kawanomoto, S. 2004, A\&A, 418, 67

Oelkers, R. J., Rodriguez, J. E., Stassun, K. G., et al. 2018, AJ, 155, 39

Ojha, D. K., Tej, A., Schultheis, M., et al. 2007, MNRAS, 381, 1219

Pashchenko, I. N., Sokolovsky, K. V., \& Gavras, P. 2018, MNRAS, 475, 2326

Payne-Gaposchkin, C. 1951, in Astrophysics, ed. J. A. Hynek (New York: McGraw-Hill), 495

Pedregosa, F., Varoquaux, G., Gramfort, A., et al. 2012, J. Mach. Learn. Res., 12, 2825, http://www.jmlr.org/papers/v12/pedregosa11a

Pepper, J., Kuhn, R. B., Siverd, R., et al. 2012, PASP, 124, 230

Pepper, J., Pogge, R. W., DePoy, D. L., et al. 2007, PASP, 119, 923

Pojmanski, G. 2002, AcA, 52, 397

Richards, J. W., Starr, D. L., Butler, N. R., et al. 2011, ApJ, 733, 10

Samus', N. N., Kazarovets, E. V., Durlevich, O. V., et al. 2017, ARep, 61, 80

Scargle, J. D. 1982, ApJ, 263, 835

Schwarzenberg-Czerny, A. 1989, MNRAS, 241, 153

Shappee, B. J., Prieto, J. L., Grupe, D., et al. 2014, ApJ, 788, 48

Skrutskie, M. F., Cutri, R. M., Stiening, R., et al. 2006, AJ, 131, 1163

Soszyński, I., Udalski, A., Szymański, M. K., et al. 2009, AcA, 59, 239

Soszyński, I., Udalski, A., Szymański, M. K., et al. 2011, AcA, 61, 217

Soszyński, I., Udalski, A., Szymański, M. K., et al. 2013, AcA, 63, 21

Stellingwerf, R. F. 1978, ApJ, 224, 953

Stetson, P. B. 1996, PASP, 108, 851

Tamuz, O., Mazeh, T., \& North, P. 2006, MNRAS, 367, 1521 
Trabucchi, M., Wood, P. R., Montalbán, J., et al. 2017, ApJ, 847, 139

Udalski, A., Szymanski, M., Kaluzny, J., et al. 1992, AcA, 42, 253

Uttenthaler, S., Blommaert, J. A. D. L., Wood, P. R., et al. 2015, MNRAS, 451,1750

VanderPlas, J. T. 2018, ApJS, 236, 16

van der Walt, S., Colbert, S. C., \& Varoquaux, G. 2011, CSE, 13, 22

Vogt, N., Contreras-Quijada, A., Fuentes-Morales, I., et al. 2016, ApJS, 227, 6

Watson, C. L., Henden, A. A., \& Price, A. 2006, SASS, 25, 47

Whitelock, P., \& Feast, M. 2000, MNRAS, 319, 759

Whitelock, P., Menzies, J., Feast, M., et al. 1994, MNRAS, 267, 711

Whitelock, P. A. 1996a, in Light Curves of Variable Stars. A Pictorial Atlas, ed. C. Sterken \& C. Jaschek (Cambridge: Cambridge Univ. Press), 106

Whitelock, P. A. 1996b, in Light Curves of Variable Stars. A Pictorial Atlas, ed. C. Sterken \& C. Jaschek (Cambridge: Cambridge Univ. Press), 98
Whitelock, P. A. 2012, Ap\&SS, 341, 123

Whitelock, P. A. 2013, in IAU Symp. 289, Advancing the Physics of Cosmic Distances, ed. R. de Grijs (Cambridge: Cambridge Univ. Press), 209

Whitelock, P. A., Feast, M. W., Marang, F., et al. 1997, MNRAS, 288, 512

Whitelock, P. A., Feast, M. W., Marang, F., et al. 2006, MNRAS, 369, 751

Whitelock, P. A., Feast, M. W., \& Van Leeuwen, F. 2008, MNRAS, 386, 313

Wood, P. R., Alcock, C., Allsman, R. A., et al. 1999, in IAU Symp. 191, Asymptotic Giant Branch Stars, ed. T. Le Bertre, A. Lebre, \& C. Waelkens (Cambridge: Cambridge Univ. Press), 151

Woźniak, P. R., Vestrand, W. T., Akerlof, C. W., et al. 2004a, AJ, 127, 2436 Woźniak, P. R., Williams, S. J., Vestrand, W. T., et al. 2004b, AJ, 128, 2965

Yuan, W., He, S., Macri, L. M., et al. 2017, AJ, 153, 170

Zacharias, N., Finch, C. T., Girard, T. M., et al. 2013, AJ, 145, 44

Zechmeister, M., \& Kürster, M. 2009, A\&A, 496, 577 